IMPERIAL/TP/98-99/45

\title{
Spacetime and the Philosophical Challenge of Quantum Gravity $^{1}$
}

\author{
J. Butterfield ${ }^{2}$ \\ All Souls' College \\ Oxford OX1 4AL \\ and \\ C.J. Isham $^{3}$ \\ The Blackett Laboratory \\ Imperial College of Science, Technology \& Medicine \\ South Kensington \\ London SW7 2BZ
}

17 March 1999

\begin{abstract}
We survey some philosophical aspects of the search for a quantum theory of gravity, emphasising how quantum gravity throws into doubt the treatment of spacetime common to the two 'ingredient theories' (quantum theory and general relativity), as a 4-dimensional manifold equipped with a Lorentzian metric. After an introduction (Section 1), we briefly review the conceptual problems of the ingredient theories (Section 2) and introduce the enterprise of quantum gravity (Section 3 ). We then describe how three main research programmes in quantum gravity treat four topics of particular importance: the scope of standard quantum theory; the nature of spacetime; spacetime diffeomorphisms, and the so-called problem of time (Section 4). By and large, these programmes accept most of the ingredient theories' treatment of spacetime, albeit with a metric with some type of quantum nature; but they also suggest that the treatment has fundamental limitations. This prompts the idea of going further: either by quantizing structures other than the metric, such as the topology; or by regarding such structures as phenomenological. We discuss this in Section 5.
\end{abstract}

\footnotetext{
${ }^{1}$ To appear in Physics meets Philosophy at the Planck Scale, ed. C. Callender and N. Huggett, Cambridge University Press (2000).

2email: jb56@cus.cam.ac.uk, jeremy.butterfield@all-souls.oxford.ac.uk

3 email: c.isham@ic.ac.uk
} 


\section{Introduction}

\subsection{Prologue}

Any branch of physics will pose various philosophical questions: for example about its concepts and general framework, and the comparison of these with analogous structures in other branches of physics. Indeed, a thoughtful consideration of any field of science leads naturally to questions within the philosophy of science.

However, in the case of quantum gravity we rapidly encounter fundamental issues that go well beyond questions within the philosophy of science in general. To explain this point, we should first note that by 'quantum gravity' we mean any approach to the problem of combining (or in some way 'reconciling') quantum theory with general relativity. ${ }^{4}$ An immense amount of effort has been devoted in the last forty years to combining these two pillars of modern physics. Yet although a great deal has been learned in the course of this endeavour, there is still no satisfactory theory: rather, there are several competing approaches, each of which faces severe problems, both technical and conceptual.

This situation means that there are three broad ways in which quantum gravity raises questions beyond the philosophy of science in general.

1. First, each of the 'ingredient theories' - quantum theory and general relativity - poses significant conceptual problems in its own right. Since several of these problems are relevant for various topics in quantum gravity (as Part 3 of this volume bears witness), we must discuss them, albeit briefly. We will do this in Section 2, to help set the stage for our study of quantum gravity. But since these problems are familiar from the literature in the philosophy, and foundations, of physics, we shall be as brief as possible.

2. Second, the fundamentally disparate bases of the two ingredient theories generate major new problems when any attempt is made to combine

\footnotetext{
${ }^{4}$ Though this construal of 'quantum gravity' is broad, we take it to exclude studies of a quantum field propagating in a spacetime manifold equipped with a fixed background Lorentzian metric. That is to say, 'quantum gravity' must involve in some way a quantum interaction of the gravitational field with itself. Quantum field theory in a fixed background is a rich subject in its own right, and it is a useful way of probing certain aspects of quantum gravity. But it does not raise the philosophical problems that we will pursue in this paper.
} 
them. This will be this paper's main focus. But even to summarize these new conceptual problems is a complicated and controversial task: complicated because these problems are closely related to one another, and to the technical problems; and controversial because what the problems are, and how they are related to one another, depends in part on problematic matters in the interpretation of the ingredient theories. Accordingly, the main task of this paper will be just to give such a summary; or rather, part of such a summary - roughly speaking, the part that relates to the treatment of spacetime. ${ }^{5}$ We undertake this task in Sections 3 et seq.

3. Third, the contrast between our lacking a satisfactory theory of quantum gravity and our having supremely successful ingredient theories, raises questions about the nature and function of philosophical discussion of quantum gravity. It clearly cannot 'take the theory as given' in the way that most philosophy of physics does; so how should it proceed? Though we are cautious about the value of pursuing meta-philosophical questions, we think this question deserves to be addressed. We do so in the course of Section 1.2; (where we also emphasise how unusual quantum gravity is, as a branch of physics).

So much by way of gesturing at the entire scope of "philosophy of quantum gravity'. In the rest of this Subsection, we shall make some brief general comments about the source of the conceptual problems of quantum gravity, and thereby lead up to a more detailed prospectus.

Despite the variety of programmes, and of controversies, in quantum gravity, most workers would agree on the following, admittedly very general, diagnosis of what is at the root of most of the conceptual problems of quantum gravity. Namely: general relativity is not just a theory of the gravitational field - in an appropriate sense, it is also a theory of spacetime itself; and hence a theory of quantum gravity must have something to say about the quantum nature of space and time. But though the phrase 'the quantum nature of space and time' is portentous, it is also very obscure, and opens up a Pandora's box of challenging notions.

To disentangle these notions, the first thing to stress is that-despite

\footnotetext{
${ }^{5}$ We choose this topic partly in the light of this volume's emphasis on spacetime, especially in Parts 2 and 5.
} 
the portentous phrase - there is, in fact, a great deal in common between the treatments of space and time given by the ingredient theories, quantum theory and general relativity. Specifically: they both treat space and time as aspects of spacetime, which is represented as a 4-dimensional differentiable manifold, while the metrical structure of spacetime is represented by a Lorentzian metric on this manifold.

In view of this, one naturally expects that a theory of quantum gravity will itself adopt this common treatment, or at least its main ingredient, the manifold conception of space and time. Indeed, we will see in Section 4 that the three main research programmes in quantum gravity do accept this conception. So returning to our phrase, 'the quantum nature of space and time': although 'quantum' might suggest 'discrete', the 'quantum nature of space and time' need not mean abandoning a manifold conception of space and time at the most fundamental level. And consequently: the clash mentioned above - between the disparate bases of the ingredient theories, quantum theory and general relativity - need not be so straightforward as the contradiction between discreteness and continuity. As we shall see in Sections 3 et seq., the clash is in fact both subtle and multi-faceted.

But we will also see in Section 4 that in various ways, and for various reasons, these programmes do not accept all of the common treatment, especially as regards the dimensionality and metric structure of spacetime: the main difference being their use of some type of quantized metric. Furthermore, the two main current programmes even suggest, in various ways, that the manifold conception of spacetime is inapplicable on the minuscule length-scales characteristic of quantum gravity.

So the situation is curious: although the ingredient theories have much in common in their treatments of space and time, this common treatment is threatened by their attempted unification. This situation prompts the idea of departing more radically from the common treatment. Besides, quite apart from the challenges to the manifold conception that come from the programmes in Section 4, other quantum gravity programmes reject this conception from the outset. We explore these ideas in Section 5.

Here it is helpful to distinguish two general strategies for going beyond the common treatment, the first more specific than the second. (1) One can quantize a classical structure which is part of that treatment, and then recover it as some sort of classical limit of the ensuing quantum theory. (2) More generally, one can regard such a structure as phenomenological, in the 
physicists' sense of being an approximation, valid only in regimes where quantum gravity effects can be neglected, to some other theory (not necessarily a quantum theory). In more philosophical jargon, this second strategy is to regard the classical structure as emergent from the other theory - though here 'emergence' must of course be understood as a relation between theories, not as a temporal process. As we will see, both these strategies can be applied either to metrical structure (as in the programmes in Section 4), or to structures required just by the manifold conception, such as topology (Section 5).

But whichever of these two strategies one adopts, there are clearly two rather different ways of thinking about the relation between the familiar treatment of space and time - in common between the ingredient theoriesand the treatment given by the, as yet unknown, theory of quantum gravity (with or without a manifold at the fundamental level). First, one can emphasise the emergence of the familiar treatment: its being 'good enough' in certain regimes. We adopt this perspective in a complementary essay. ${ }^{6}$ Second, one can emphasise instead 'the error of its ways'. That is, one can emphasise how quantum gravity suggests limitations of the familiar treatment; (though the conclusions of the examination will of course be tentative, since we have no satisfactory theory of quantum gravity). That is the perspective of this paper. $^{7}$

In more detail, our plan is as follows. First we make some general orienting remarks about philosophy of quantum gravity (Section 1.2) and realism (Section 1.3). Then we briefly review the bearing on quantum gravity of the conceptual problems of the ingredient theories: quantum theory (Section 2.1), and more briefly, general relativity (Section 2.2). In Section 3, we introduce the enterprise of quantum gravity proper. We state some of the main approaches to the subject; summarise some of the main motivations for

\footnotetext{
${ }^{6}$ See [1]. It discusses philosophical aspects of emergence, which here we only treat very briefly, in Section 5. But in another regard, it is more limited: it discusses the emergence of spatio-temporal concepts for only one of the three programmes in Section 4.

${ }^{7}$ We should add that though this paper focusses on the treatment of spacetime, quantum gravity also 'puts pressure' on the formalism of, and usual interpretative ideas about, quantum theory; as we shall briefly discuss in Section 2. Furthermore, quantum gravity even puts pressure on standard mathematics itself, in that constructing a theory of quantum gravity might require some non-standard mathematical ideas: for example, spacetime might be represented by something that is not a set. We will briefly discuss this line of thought in Section 5 .
} 
studying it (Section 3.1); and introduce some of the conceptual aspects that relate closely to spacetime: the role of diffeomorphisms (Section 3.2) and the problem of time (Section 3.3).

In Section 4, we first set up the four topics in terms of which we will survey three well-developed research programmes in quantum gravity. The topics are: (i) the extent to which the programme uses standard quantum theory; (ii) the extent to which it uses standard spacetime concepts; (iii) how it treats spacetime diffeomorphisms; and (iv) how it treats the problem of time. After some historical remarks (Section 4.2), we turn to our three research programmes: the old particle-physics approach (Section 4.3), supergravity and superstrings (Section 4.4), and canonical quantum gravity (Section 4.5). (We also briefly treat a distinctive version of the latter, the Euclidean programme.) By and large, these three programmes are 'conservative' in the sense that although they in some way quantize metric structure, they use standard quantum theory, and they treat the spacetime manifold in a standard way. But in various ways, they also suggest that this treatment of spacetime is phenomenological. To mention two obvious examples: in the superstring programme, there are suggestions that many different manifolds play a role. And in the main current version of canonical quantum gravity (the Ashtekar programme), there are suggestions that quantities such as area and volume are quantised; and that the underlying structure of spacelike may be more like a combinatorial network than a standard continuum manifold.

This situation prompts the idea of a quantum gravity programme that $a b$ initio goes beyond the standard treatments of spacetime and/or quantum theory. So in Section 5, we discuss some of these more radical ideas: quantizing spacetime structures other than the metric, or regarding such structures as phenomenological - where this could involve abandoning the manifold conception in favour of a variety of novel mathematical structures.

Finally, a caveat about the scope of the paper. Even for the well-established research programmes, we shall omit, or mention only in passing, many of the more recent ideas. This is partly because of lack of space, and partly because technical ideas need to acquire a certain degree of maturity before philosophical reflection becomes appropriate. Similarly, we will not mention many of the more speculative ideas that have emerged over the years. Instead, we have deliberately limited ourselves to some of the well-established ideas in a few of the well-established research programmes, and to just a few of the more speculative ideas. 


\subsection{No Data, No Theory, No Philosophy?}

Before embarking on this detailed analysis of the conceptual challenge of quantum gravity, it is important to briefly consider the general idea of philosophical discussion of quantum gravity: more pointedly, to defend the idea, in the face of the fact that there is far from being a universally agreed theory of quantum gravity! So in this Subsection, we will point out the peculiarity of quantum gravity in comparison with other branches of theoretical physics, and discuss how this affects the way in which one can write about the subject from a philosophical perspective. This will lead to some discussion of the philosophical positions we intend to adopt, especially as regards realism.

The most obvious peculiarity is a dire lack of data. That is, there are no phenomena that can be identified unequivocally as the result of an interplay between general relativity and quantum theory - a feature that arguably challenges the right of quantum gravity to be considered as a genuine branch of science at all!

This lack of obvious empirical data stems from a simple dimensional argument that quantum gravity has a natural length scale - the Planck length defined using dimensional analysis as ${ }^{8} L_{P}:=\left(G \hbar / c^{3}\right)^{\frac{1}{2}}$ — and this is extremely small: namely $10^{-35} \mathrm{~m}$. By comparison: the diameters of an atom, proton and quark are, respectively, about $10^{-10} \mathrm{~m}, 10^{-15} \mathrm{~m}$, and $10^{-18} \mathrm{~m}$. So the Planck length is as many orders of magnitude from the (upper limit for) the diameter of a quark, as that diameter is from our familiar scale of a metre! The other so-called 'Planck scales', such as the Planck energy and the Planck time, are equally extreme: the Planck energy $E_{P}$ has a value of $10^{22} \mathrm{MeV}$, which is far beyond the range of any foreseeable laboratory-based experiments; and the Planck time (defined as $T_{P}:=L_{P} / c$ ) has a value of about $10^{-42}$ seconds.

These values suggest that the only physical regime where effects of quantum gravity might be studied directly - in the sense that something very specific can be expected - is in the immediate post big-bang era of the universewhich is not the easiest thing to probe experimentally! This problem is compounded by the fact that, modulo certain technical niceties, any Lorentzinvariant theory of interacting spin-2 gravitons with a conserved energymomentum tensor will yield the same low-energy scattering amplitudes as those obtained from a perturbative expansion of the Einstein Lagrangian. Thus different quantum gravity theories might only reveal their differences

\footnotetext{
${ }^{8}$ Here $G$ is Newton's constant, $\hbar$ is Planck's constant, and $c$ is the speed of light.
} 
empirically at very high energies.

This lack of data has implications for both physics and philosophy. For physics, the main consequence is simply that it becomes very difficult to build a theory: witness the fact that a fully satisfactory quantum theory of gravity remains elusive after forty years of intense effort. Of course, a great deal has been learnt about the features that such a theory might possess, albeit partly by eliminating features that are now known not to work. A good example is the perturbative unrenormalizability of the particle-physics approach to quantum gravity (see Section 4.3).

But there is more to the difficulty of theory-construction than just the lack of data: and this 'more' relates to philosophy in two ways. Firstly, this difficulty is partly due to conceptual problems which clearly bear on philosophical discussions of concepts such as space, time and matter. Here we have in mind both kinds of conceptual problem, listed as 1. and 2. in Section 1.1: those arising from the disparateness of the bases of general relativity and quantum theory, and also problems about each of these theories in themselves. To take an obvious example of the latter, quantum gravity is usually taken to include quantum cosmology; and here, the idea of a 'quantum state of the universe' immediately confronts conceptual problems about quantum theory such as the meaning of probability, and the interpretation of the quantum state of a closed system - in this case, the universe in its entirety. ${ }^{9}$ And as we shall see in Section 2.2, such examples do not only come from quantum theory, with its notorious conceptual problems. They also come from general relativity - whose foundations are murkier than philosophers commonly take them to be. To sum up: the difficulty of theory-construction is partly due to conceptual problems (and is thereby related to philosophy), not just lack of data.

Secondly, theory-construction is difficult because there is not even agreement on what sorts of data a quantum theory of gravity would yield, if only we could get access to them! More precisely, the dimensional argument discussed above suggests that only phenomena at these very small distances, or high energies, would exhibit quantum gravity effects; which implies that

\footnotetext{
${ }^{9}$ There is another connection with the conceptual problems about quantum theory. Namely, the lack of data in quantum gravity research is analogous to that which-until relatively recently - faced research in conceptual problems about quantum theory. In view of these connections, it is curious that there has been so little interaction between the two research communities.
} 
the main application of the quantum theory of gravity may be to the physics of the very early universe. However, this argument rests on the assumption that any physically measurable quantity can be expressed as a power series in a number like $E / E_{P}$ (where $E$ is some characteristic energy scale for an experiment), so that the quantity's predicted value is tiny in any experiment that probes energy-scales that are much lower than $E_{P}$. But experience elsewhere in quantum field theory suggests that 'non-perturbative effects' could also occur, in which the predicted values of certain measurable quantities are not analytic functions of the coupling constant, and this totally changes the argument: for example, if $x$ is a very small number, then $|\log x|$ is very large! But, in the case of quantum gravity it is anybody's guess what sort of effects would be exhibited - and therefore, what sort of predictions the envisaged quantum theory of gravity is meant to give. For example, for all we know, it might predict the masses of all the elementary particles.

The most obvious consequence of such uncertainty is that it makes for a sort of circularity. On the one hand, it is ferociously difficult to find the theory without the help of data, or even an agreed conception of the sort of data that would be relevant. On the other hand, we can only apply our present theories to (and get evidence from) regimes well way from those determined by the Planck scale; so we cannot judge what phenomena might be relevant to a theory of quantum gravity, until we know what the theory is.

In this predicament, theory construction inevitably becomes much more strongly influenced by broad theoretical considerations, than in mainstream areas of physics. More precisely, it tends to be based on various prima facie views about what the theory should look like - these being grounded partly on the philosophical prejudices of the researcher concerned, and partly on the existence of mathematical techniques that have been successful in what are deemed, perhaps erroneously, to be closely related areas of theoretical physics, such as non-abelian gauge theories. In such circumstances, the goal of a research programme tends towards the construction of abstract theoretical schemes that are compatible with some preconceived conceptual framework, and are internally consistent in a mathematical sense.

This situation does not just result in an extreme 'underdetermination of theory by data', in which many theories or schemes, not just a unique one, are presented for philosophical assessment. More problematically, it tends to produce schemes based on a wide range of philosophical motivations, which 
(since they are rarely articulated) might be presumed to be unconscious projections of the cthonic psyche of the individual researcher - and might be dismissed as such! Indeed, practitioners of a given research programme frequently have difficulty in understanding, or ascribing validity to, what members of a rival programme are trying to do. This is one reason why it is important to uncover as many as possible of the assumptions that lie behind each approach: one person's 'deep' problem may seem irrelevant to another, simply because the starting positions are so different.

This situation also underlines the importance of trying to find some area of physics in which any putative theory could be tested directly. A particularly important question in this context is whether the dimensional argument discussed above can be overcome, i.e., whether there are measurable quantum gravity effects well below the Planck scales; presumably arising from some sort of non-perturbative effect. However, the existence of such effects, and the kind of phenomena which they predict, are themselves likely to be strongly theory-dependent.

It follows from all this that the subject of quantum gravity does not present the philosopher with a conceptually or methodologically unified branch of physics, let alone a well-defined theory; but instead with a wide and disparate range of approaches. We must turn now to discussing what this situation implies about the scope and possible topics of philosophical discussion.

As we conceive it, philosophy of physics is usually concerned with either: (i) metaphysical/ontological issues, such as the nature of space, or physical probability; or (ii) epistemological/methodological issues, such as underdetermination of theories, or scientific realism, with special reference to physics. And in both areas (i) and (ii), the discussion is usually held in focus by restricting itself to a reasonably well-defined and well-established physical theory; or at worst, to a small and homogeneous set of reasonably well-defined rival theories. Hence the image of philosophy of physics as a Greek chorus, commenting post facto on the dramatic action taking place on a well-defined stage. But, as we have seen, this kind of role is not available in quantum gravity, where there are no reasonably well-defined, let alone wellestablished, theories. So should philosophical discussion simply wait until the subject of quantum gravity is much better established?

We think not. For there are at least three (albeit overlapping) ways in which to pursue the subject 'philosophy of quantum gravity'; each of them valuable, and each encompassing many possible projects. The first two are 
straightforward in that all will agree that they are coherent endeavours; the third is more problematic. This paper will be an example of the second way. Broadly speaking, they are, in increasing order of radicalism:

1. First, one can undertake the 'normal' sort of philosophical analysis of some sufficiently specific and well-defined piece of research in quantum gravity; even though the price of its being well-defined may be that it is too specific/narrow to warrant the name 'theory' - so that, in particular, it would be unwise to give much credence to the ontological or interpretative claims it suggests. An example might be one of the specific perturbative approaches to string theory - for example, the type II-B superstring - which recent research in the area suggests might merely be one particular perturbative regime, of an underlying theory (' $M$-theory') whose mathematical and conceptual structures are quite different from those of a quantised loop propagating in a spacetime manifold. This sort of analysis is certainly valuable, even if the limitations of any specific example must make one wary of any ontological or interpretative suggestions which arise. For examples of such analyses, see the papers in Parts 3 and 4 of this volume.

2. Second: one can try to relate a range of conceptual problems about general relativity, quantum theory, and their having disparate bases, to a range of approaches or research programmes in quantum gravity.

To make this endeavour manageable, one must inevitably operate at a less detailed level than in 1. above. The hope is that despite the loss of detail, there will be a compensating value in seeing the overall pattern of relationships between conceptual problems and mathematical/physical approaches to quantum gravity. Indeed, one can hope that such a pattern will be illuminating, precisely because it is not tied to details of some specific programme that may be on the proverbial hiding to nowhere.

Such a pattern of relationships can be envisioned in two ways, according to which 'side' one thinks of as constraining the other. One can think of problems and ideas about general relativity and quantum theory as giving constraints on - or heuristic guides to our search forquantum gravity. (Of course, we harbour no illusions that such conceptual discussion is a prerequisite for successful theory-construction-of 
that, only time will tell.) And vice versa, one can think of quantum gravity as constraining those problems and ideas, and even as suggesting possible changes to the foundations of these constituent theories. In what follows, we shall see examples of both kinds of constraint.

3. Third, one can try to study quantum gravity in the context of some traditional philosophical ideas that have nothing to do with the interpretation of general relativity and quantum theory per se-for example, traditional concepts of substance and attribute. Again, one can think of such a relation in two ways: the philosophical idea giving constraints on quantum gravity; and vice versa, quantum gravity reflecting back on the philosophical idea.

We admit to finding this endeavour alluring. But, again, we harbour no illusions that such traditional philosophical ideas are likely to be heuristically helpful, let alone a prerequisite, for theory-construction. Similarly, one must be tentative about constraints in the opposite direction; i.e. about the idea that a traditional philosophical position could be 'knocked out' by a quantum gravity proposal, where 'knocked out' means that the position is shown to be, if not false, at least 'merely' phenomenological, or approximately true, in ways that philosophers tend not to realize is on the cards. Since no quantum gravity proposal is well-established, any such 'knock-out' is tentative.

This paper will exemplify type 2. above, although we emphasise that we are sympathetic to type 3. More generally, we are inclined to think that in the search for a satisfactory theory of quantum gravity, a fundamental reappraisal of our standard concepts of space, time and matter may well be a necessary preliminary. Thus we are sceptical of the widespread idea that at the present stage of quantum gravity research, it is better to try first to construct an internally consistent mathematical model and only then to worry about what it 'means'. But such a reappraisal is fiercely hard to undertake; and accordingly, this paper adopts 'the middle way' - type 2.

A final remark about the various ways to pursue 'philosophy of quantum gravity'. Though type 2. involves, by definition, surveying ideas and approaches, this by no means implies that 2. encompasses a single project, or even that it encompasses only one project focussing on the nature of space and time. For example, here is an alternative project, relating to traditional 
positions in the philosophy of geometry.

The immense developments in pure and physical geometry from Riemann's habilitationschrift of 1854 to the establishment of general relativity, transformed the philosophy of geometry beyond recognition. In particular, Kant's apriorism fell by the wayside, to be replaced by empiricism and conventionalism of various stripes. With this transformation, the idea that at very small length-scales, space might have a non-manifold structure became a 'live option' in a way that it could not have been while Kant's influence held sway in its original form. Yet, in fact, this idea has had only a small role in the philosophy of geometry of the last 150 years - for the perfectly good reason that no significant physical theory took it up. (Its main role is via Riemann's view - endorsed in our own day by Grunbaum - that in a discrete space, but not a manifold, the metric is, or can be, intrinsic, and thereby non-conventional.)

But nowadays, there are several 'unconventional' approaches to quantum gravity that postulate a non-manifold structure for spacetime; and even in the more conventional approaches, which do model space, or spacetime, with a differentiable manifold, there are often hints of a discrete structure that lies beneath the continuum picture with which one starts. For example, in the Ashtekar programme, area and volume variables become discrete; and in superstring theory there are strong indications that there is a minimal size for length.

These proposals prompt many questions for philosophers of geometry; the obvious main one being, how well can the traditional positions - the various versions of empiricism and conventionalism - accommodate such proposals? We will not take up such questions here; though we like to think that this paper's survey of issues will help philosophers to address them.

\subsection{Realism?}

Finally, we should briefly discuss the bearing of our discussion on the fundamental questions of realism. Thus it is natural to ask us (as one might any authors in the philosophy of physics):-Does the discussion count for or against realism, in particular scientific realism; or does it perhaps presuppose realism, or instead its falsity?

Our answer to this is broadly as follows. We will write as if we take proposals in quantum gravity realistically, but in fact our discussion will not 
count in favour of scientific realism-nor indeed, against it. This lack of commitment is hardly surprising, if only because, as emphasised in Section 1.2, quantum gravity is too problematic as a scientific field, to be a reliable test-bed for scientific realism. But we will fill out this answer in the rest of this Subsection. In short, we will claim: i) we are not committed to scientific realism; and ii) there is a specific reason to be wary of reifying the mathematical objects postulated by the mathematical models of theoretical physics. We shall also make a comment relating to transcendental idealism.

\subsubsection{Beware Scientific Realism}

Scientific realism says, roughly speaking, that the theoretical claims of a successful, or a mature, scientific theory are true or approximately true, in a correspondence sense, of a reality independent of us. So it is a conjunctive thesis, with an ontological conjunct about the notion of truth as correspondence, and an independent reality; and an epistemic conjunct of 'optimism'-about our mature theories 'living up' to the first conjunct's notions.

Obviously, discussions of quantum gravity (of any of the three types of Section 1.2) need not be committed to such a doctrine, simply because, whatever exactly 'successful' and 'mature' mean, quantum gravity hardly supplies us with such theories! That is: even if one endorses the first conjunct of scientific realism, the second conjunct does not apply to quantum gravity. So there is no commitment, whatever one's view of the first conjunct.

But there is another way in which we might seem to be committed to scientific realism: namely, through our treatment of the 'ingredient theories', quantum theory and general relativity. In Section 2 et seq. we will often write about the interpretation of these theories, in an ontological (rather than epistemological or methodological) sense; as does much current work in the philosophy of physics. For example, we will mention the so-called 'interpretations' of quantum theory (Copenhagen, Everettian, pilot-wave etc.); which are in fact ontologies, or world-views, which the philosopher of quantum theory elaborates and evaluates. Similarly as regards general relativity; for example, we will sometimes write about the existence of spacetime points as if they were objects.

But it should not be inferred from our writing about these topics in this way that we are committed to some form of scientific realism. There is no such entailment; for two reasons, one relating to each of scientific realism's 
two conjuncts. The first, obvious reason concerns the epistemic optimism of scientific realism. Clearly, elaborating and evaluating ontologies suggested by scientific theories is quite compatible with denying this optimism.

The second reason is perhaps less obvious. We maintain that such elaboration and evaluation of ontologies involves no commitment to a correspondence notion of truth, or approximate truth, characteristic of realism. This can seem surprising since for philosophers of physics, 'electron' and 'spacetime point' come as trippingly off the tongue, as 'chair' and other words for Austin's 'medium-sized dry goods' come off all our tongues, in everyday life. And this suggests that these philosophers' account of reference and truth about such topics as electrons is as realist, as is the account by the so-called 'commonsense realist' of reference to chairs, and of the truth of propositions about chairs. But the suggestion is clearly false. Whatever general arguments (for example, about ontological relativity) can be given against realist accounts of reference and truth in regard to chairs (and of course, rabbits and cats - Quine's and Putnam's 'medium-sized wet goods'!) can no doubt also be applied to electrons and spacetime points. Indeed, if there is to be a difference, one expects them to apply with greater, not lesser, force; not least because - at least, in the case of the electron - of the notorious difficulty in understanding a quantum 'thing' in any simple realist way.

Here we should add that in our experience, philosophers of physics do in fact tend to endorse realist accounts of reference and truth. We suspect that the main cause of this is the powerful psychological tendency to take there to be real physical objects, corresponding in their properties and relations to the mathematical objects in mathematical models, especially when those models are very successful. But this tendency is a cause, not a reason; i.e., it does not support the suggestion we denied above, that elaborating a physical theory's ontology implies commitment to realism. Whitehead had a vivid phrase for this tendency to reification: 'the fallacy of misplaced concreteness'.

For this paper, the main example of this psychological urge will be the tendency to reify spacetime points, which we shall discuss in more detail in Section 2.2. For now, we want just to make three general points about this tendency to reification. The third is more substantial, and so we devote the next Subsection to it.

First, such reification is of course a common syndrome in the praxis of physics, and indeed the rest of science; carried over, no doubt, from an excessive zeal for realism about say, chairs, in everyday life. Certainly, in 
so far as they take a view on these matters, the great majority of physicists tend to be straightforward realists when referring to electrons, or even such exotic entities as quarks.

Second, reification is not just a psychological tendency, or a pedagogic crutch. It can also be heuristically fruitful, as shown by successful physical prediction based on the mathematics of a theory; for example, Dirac's prediction of the positron as a 'hole' in his negative energy 'sea' (though he at first identified the holes with protons!).

\subsubsection{The Fragility of Ontology in Physics}

Setting aside our general cautiousness about scientific realism, there is a specific reason to be wary of misplaced concreteness in theoretical physics. We cannot develop it fully here, but we must state it; for it applies in particular to such putative objects as spacetime points, which will of course be centre-stage in this paper.

The reason arises from the idea that physics aims to supply a complete description of its subject-matter. It does not matter how exactly this idea is made precise: for example, what exactly 'complete description' means, and whether this aim is part of what we mean by 'physics'. The rough idea of physics aiming to be complete is enough. For it entails that in physics, or at least theoretical physics, a change of doctrine about a subject-matter is more plausibly construed as a change of subject-matter itself, than is the case in other sciences. So in physics (at least theoretical physics), old ontologies are more liable to be rejected in the light of new doctrine.

We can make the point with a common-sense example. Consider some body of common-sense doctrine, say about a specific table, or tables in general. Not only is it fallible - it might get the colour of the specific table wrong, or it might falsely say that all tables have four legs - it is also bound to be incomplete, since there will be many facts that it does not includefacts which it is the business of the special sciences, or other disciplines, to investigate; for example, the material science of wood, or the history of the table(s). Similarly for a body of doctrine, not from common-sense, but from a science or discipline such as chemistry or history, about any subject-matter, be it wood or the Napoleonic wars. There are always further facts about the subject-matter, not included in the doctrine. Indeed this is so, even if the body of doctrine is the conjunction of all the facts about the subject-matter 
expressible in the taxonomy (vocabulary) of the discipline concerned. Even for such a giant conjunction, no enthusiast of such a science or discipline is mad enough, or imperialist enough, to believe that it gives a complete description of its subject matter. There are always other conjuncts to be had, typically from other disciplines.

Not so, we submit, for physics, or at least theoretical physics: whether it be madness, imperialism, or part of what we mean by 'physics', physics does aspire to give just this sort of complete description of its subject-matter. And this implies that when 'other conjuncts arrive'-i.e., new facts come to light additional to those given by the most complete available physical description - it is more reasonable to construe the new facts as involving a change of subject-matter, rather than as an additional piece of doctrine about the old subject-matter.

Note that we do not say that the first construal is always more reasonable than the second - by no means! Only that it is usually more reasonable than in other sciences, simply because of physics' aspiration to completeness. To take an obvious example: the very fact that a quantum-theoretic description of the electron before the discovery of spin aspired to be complete, makes it more reasonable to construe the discovery of the magnetic moment of the electron as a change of subject-matter - the replacement of the 'old' ontology comprising the spinless electron, by one with a spinning electron - rather than as just additional doctrine about the old subject-matter, the 'old electron'. To sum up this point: the fact that physics aspires to give a complete description of its subject-matter gives a specific reason to be wary of reifying the objects postulated by physical theories. ${ }^{10}$

\subsubsection{The Question of Transcendental Idealism}

Any discussion about realism - even one mainly concerned, as we are, with scientific realism-raises the issue of transcendental idealism: that is, as

\footnotetext{
${ }^{10}$ As mentioned, lack of space prevents a full defence of this point. Suffice it here to add two comments. (1) The point does not assume that new doctrine which does not change the subject-matter must be cumulative, i.e., must not contradict old doctrine. Suppose doctrine can be withdrawn or adjusted, without the subject-matter changing: the point still holds good. (2) The point is independent of physicalism. For it turns on physics aspiring to give a complete description of its subject-matter. But this implies nothing about whether the subject-matter of physics exhausts all (empirical) subject-matters, i.e., about whether physicalism is true.
} 
we understand it, the issue whether there is, or must be thought to be, something beyond the 'appearances' - or in more modern terms, the 'ontology of science'. This issue comes to mind all the more readily in a discussion of realism concerning space and time, since it was in connection with these categories that Kant forged his transcendental idealism.

Of course we have no space here to address this enormous issue. ${ }^{11}$ Instead, we confine ourselves to two short remarks. First: Like most authors in the philosophy of physics, and almost all theoretical physicists ${ }^{12}$, we will write for convenience and brevity 'as if' transcendental realism is true: more precisely, as if there is nothing beyond 'the ontology of science'. But we stress that 'the ontology of science' need not be understood in terms of the claims of our best, or a mature, scientific theory. For example, the phrase can be understood in terms of a Peircean limit of enquiry; with no implication that our best, or mature, scientific theories approximate this limit. So whether or not transcendental realism is true, it is in any case consistent with rejecting scientific realism.

Second, a remark specific to quantum gravity that relates to the minuscule size of the Planck length, emphasised at the start of Section 1.2. Namely, it is so minuscule as to suggest that those aspects of reality that require a theory of quantum gravity for their description do not deserve such names as 'appearance', 'phenomenon', or 'empirical'. Agreed, there is no hint in the writings of Kant or other Kantians that one should restrict the word 'appearance' to what is practically accessible. And one naturally thinks that an 'item' (event, state of affairs, call it what you will) that is localized in spacetime, or that somehow has aspects localized in spacetime, is ipso facto an appearance, part of empirical reality-be it, or its aspects, ever so small. But we would like to suggest that one should resist this, and to consider taking the inaccessibility of these scales of length, energy etc. to be so extreme as to be truly 'in principle'. To put the point in terms of 'empirical': the suggestion is that these items, or their localized aspects, are not empirical, though one might still call them 'physical', as well as, of course, 'real' and 'actual' - in particular, these items or aspects would be represented in our

\footnotetext{
${ }^{11}$ After all, even if one confines oneself to the topic of space and time, and to authors who seem to deserve the name 'transcendental idealists', there are several positions to evaluate; for example, Kant's view of space and time as ideal - the a priori conditions for all experience - and Kuhn's view of the essential reality of space.

${ }^{12}$ In fact, the theoretical physicist (CJI) in this collaboration does not hold this view.
} 
theory of quantum gravity. ${ }^{13}$

If this is right, one could perhaps reconcile various Kantian claims that space and time must have some features - for example, being continua - as an a priori matter with the claims of those quantum gravity programmes that deny space and time those features. The apparent contradiction would be an artefact of an ambiguity in 'space and time': the quantum gravity programmes would not be about space and time in the Kantian sense. Finally, we should emphasize that in envisaging such a reconciliation, we are not trying to defend specific Kantian claims, such as its being an a priori matter that space and time are continua; or that geometry is Euclidean. Indeed, we join most physicists in being sceptical of such specific claims, not least because the history of physics gives remarkable examples of the creative, albeit fallible, forging of new concepts. But we are sympathetic to the broader Kantian idea that human understanding of reality must, as an $a$ priori matter, involve certain notions of space and time.

\section{Conceptual Problems of Quantum Theory and General Relativity}

As discussed in Section 1.1, the over-arching question of this paper is: What part (if any) of the ingredient theories' common treatment of spacetime-i.e., as a differentiable manifold with a Lorentzian metric - needs to be given up in quantum gravity? It is already clear (sad to say!) that there is no agreement about the answer to this question. As we shall see in more detail in Sections 3 et seq., there is a wide variety of different quantum gravity programmes, giving different answers. And more confusingly, these different answers do not always represent simple disagreements between the programmes: sometimes two programmes are aiming to do such very different things, that their different answers need not contradict each other. Hence this paper's project of undertaking a survey.

But as we shall also see, this variety of programmes, and of aims, is due in part to the fact that significant conceptual problems about the ingredient

\footnotetext{
${ }^{13}$ The suggestion also allows what we hoped for in Section 1.2: namely, quantum gravity effects at much more accessible length scales; it only contends that aspects we cannot thus probe are not empirical.
} 
theories are still unsolved: both problems about the nature of quantum reality, and problems about the nature of space and time - in part, traditional philosophical problems, though of course modified in the light of general relativity and quantum theory. So it will help to set the stage for our survey, to devote this Section to describing such problems. Of course, we cannot give a thorough discussion, or even an agreed complete list, of these problems. ${ }^{14}$ We confine ourselves to briefly discussing some issues that are specifically related to quantum gravity.

In this discussion, we will place the emphasise on problems of quantum theory, for two reasons; only the second of which concerns quantum gravity. First, we agree with the 'folklore' in the philosophy of physics that quantum theory faces more, and worse, conceptual problems than does general relativity. In a nutshell: general relativity is a classical field theory (of gravitation); and broadly speaking, such theories are not mysterious, and their interpretation is not controversial. On the other hand, quantum theory is mysterious, and its interpretation is controversial. This is attested not only by the struggles of its founding fathers; but also with the ongoing struggles with issues such as the non-Boolean structure of the set of properties of a physical system, the lack of values for quantities associated with superpositions, the phenomenon of quantum entanglement - and of course, the 'confluence' of these three issues in the 'measurement problem'. Indeed, thanks to these struggles, the issues are not nearly so intractable as they were 70 , or even 40 , years ago. Though mystery remains, there are nowadays several flourishing schools of thought about how to interpret quantum theory: we will mention some of them in Section 2.1 below.

Second, as we shall see in Section 4: despite general relativity's 'merits' of interpretative clarity over quantum theory, the main quantum gravity programmes tend to put much more pressure on the framework of standard general relativity, and thus on spacetime concepts, than they do on quantum theory. Like most other research programmes using quantum theory, they simply use the standard quantum theoretic formalism, and do not address its conceptual problems. It is this disparity that motivates this paper's choice of spacetime as the main topic of its survey. But arguably, this acceptance of standard quantum theory is a mistake, for two reasons. First: in general, it would seem wise for a research programme that aims to combine, or some-

\footnotetext{
${ }^{14}$ For more discussion, see Parts 3 and 5 of this volume.
} 
how reconcile, two theories, to rely more heavily on the clearer ingredient theory, than on the mistier one! Second: as we shall see in Section 2.1, it turns out that in various ways, the search for a quantum theory of gravity raises the conceptual problems of quantum theory in a particularly acute form - and even puts some pressure on its mathematical formalism. In any case - whether or not this acceptance of quantum theory is a mistake - in this Section we will briefly 'redress the balance'. That is to say, we will emphasise the pressure that quantum gravity puts on quantum theory. ${ }^{15}$

Accordingly, our plan will be to discuss first (in Section 2.1) the conceptual problems of quantum theory, especially in relation to quantum gravity; and then the conceptual problems of general relativity (in a shorter Section 2.2).

\subsection{Interpreting Quantum Theory}

In this Subsection, our strategy will be to distinguish four main approaches to interpreting quantum theory, in order of increasing radicalism; and to show how each relates to topics, or even specific approaches, in quantum gravity. The first two approaches (discussed in Sections 2.1.1 and 2.1.2) are both conservative about the quantum formalism - they introduce no new equations. But they differ as to whether they are 'cautious' or 'enthusiastic' about the interpretative peculiarities of quantum theory. The third and fourth approaches (discussed in Sections 2.1.3 and 2.1.4) do introduce new equations; but in various (different) ways remain close enough to standard quantum theory to be called 'interpretations' of it.

We stress that although our catalogue of four approaches is by no means maverick, we make no claim that it is the best, let alone the only, way to classify the various, and complexly inter-related, interpretations of quantum theory. But of course we believe that with other such classifications, we could make much the same points about the connections between interpreting quantum theory and quantum gravity.

On the other hand, we cannot consider the details of individual interpretations within each approach. And in view of the paper's overall project, we shall emphasise how the interpretations we do mention relate to space and time - at the expense of other aspects of the interpretation. For example,

\footnotetext{
${ }^{15}$ For more such pressure, see Part 3 of this volume.
} 
we will not mention even such basic aspects as whether the interpretation is deterministic - except in so far as such aspects relate to space and time.

\subsubsection{Instrumentalism}

We dub our first approach to interpreting quantum theory, 'instrumentalism'. We intend it as a broad church. It includes views that apply to quantum theory some general instrumentalism about all scientific theories; and views that advocate instrumentalism only about quantum theory, based on special considerations about that subject. We will not comment on the first group, since we see no special connections with quantum gravity. Or more precisely, we see no connections other than those which we already adumbrated from another perspective, that of realism, in Section 1, especially Section 1.3.

On the other hand, some views in the second group do have connections with quantum gravity, albeit 'negative' ones. Thus consider the Copenhagen interpretation of quantum theory: understood, not just as the minimal statistical interpretation of the quantum formalism in terms of frequencies of measurement results, but as insisting on a classical realm external to the quantum system, with a firm 'cut' between them, and with no quantum description of the former. In so far as this classical realm is normally taken to include classical space and time ${ }^{16}$, this suggests that, in talking about 'quantum gravity', we are making a category error by trying to apply quantum theory to something that forms part of the classical background of that theory: "what God has put assunder, let no man bring together". We shall say more later about the view that a quantum theory of gravity should, or can, be avoided (Section 3.1.2). But for the most part we will accept that serious attempts should be made to construct a 'quantum theory of space and time' (or, at least, of certain aspects of space and time); with the understanding that, in doing so, it may be necessary to radically change the interpretationand, perhaps, the mathematical formalism - of quantum theory itself.

In endeavouring to interpret quantum theory, regardless of quantum gravity, this second group of views is notoriously problematic. It is not just a matter of it being difficult to understand, or to defend, Bohr's own views, or views similar to his. There are quite general problems, as follows. Any view

\footnotetext{
${ }^{16}$ Kantian themes about the a priori nature of space and time arise here, just as in Section 1.3; for Kantian aspects of Bohr, see [2].
} 
that counts as 'instrumentalism specifically about quantum theory' (i.e., any view in this group) must presumably do either or both of the following:

(i) deny that the quantum state describes individual systems, at least between measurements; or in some similar way, it must be very cautious about the quantum description of such systems;

(ii) postulate a 'non-quantum' realm, whose description can be taken literally (i.e., not instrumentalistically, as in (i)); usually this realm is postulated to be 'the classical realm', understood as macroscopic, and/or the domain of 'measurement results', and/or described by classical physics.

But recent successful applications of quantum theory to individual microphysical systems (such as atoms in a trap), and to macroscopic systems (such as superconducting squids) have made both (i) and (ii) problematic. This suggests in particular that we should seek an interpretation in which no fundamental role is ascribed to 'measurement', understood as an operation external to the domain of the formalism; see the next Subsection.

\subsubsection{Literalism}

Like instrumentalism, we intend this approach to be a broad church. The idea is to make the interpretation of quantum theory as 'close' as possible to the quantum formalism. (Hence the name 'literalism'; 'realism' would also be a good name, were it not for its applying equally well to our third and fourth approaches.) In particular, one rejects the use of a primitive notion of measurement, and associated ideas such as a special 'classical realm', or 'external observer' that is denied a quantum-theoretic description. Rather, one 'cuts the interpretation to suit the cloth of the formalism'; revising, if necessary, traditional philosophical opinions, in order to do so. Hence our remark at the start of Section 2.1 that this approach is 'enthusiastic' about the interpretative peculiarities of quantum theory, while instrumentalism is 'cautious'.

As we see it, there are two main types of literalist view: Everettian views, and those based on quantum logic. Of these types, the first has been much discussed in connection with quantum gravity (especially quantum cosmol- 
ogy); but the second, hardly at all in this connection. Accordingly, we shall only treat the first. ${ }^{17}$

As usually presented, the main aim of an Everettian view (or, as it is sometimes called these days, a 'post-Everrett' view) is to solve the 'measurement problem': i.e., the threat that at the end of a measurement, macroscopic objects (such as an instrument pointer) will have no definite values for familiar quantities like position - contrary to our experience. More specifically, the aim is to solve this problem without invoking a collapse of the state vector, or an external observer. This involves (i) resolving the state vector of a closed system as a superposition of eigenstates of a 'preferred quantity'; (ii) interpreting each of the components as representing definite positions for pointers and other macroscopic objects; and then (iii) arguing that, although there is no collapse, you will only 'see' one component in the superposition.

This summary description leaves open some crucial questions. For example:

(a) How is the preferred quantity to be chosen? Should it be in terms of familiar quantities such as position of macroscopic objects, so that each summand secures a definite macroscopic realm ('many worlds'); or should it involve arcane quantities concerning brains, whose eigenstates correspond to experiences of a definite macroscopic realm ('many minds')?

(b) Should one say that for each component there is a physically real 'branch', not just the possibility of one?

(c) How should one justify the claim that you will not 'see the other components': by some process of 'splitting of the branches', or by appeal to decoherence making the interference terms that are characteristic of the presence of other components, negligibly small?

We do not need to discuss these issues here, which have been much debated in

\footnotetext{
${ }^{17}$ About the second, suffice it to say that such views propose to revise the logic of discourse about quantum systems; (so we do not intend the type to include purely technical investigations of non-Boolean structures). But it is unclear how such proposals solve the interpretative problems of quantum theory, such as the measurement problem or nonlocality; and indeed, these proposals now seem to have few advocates - at least compared with 25 years ago. In any case, their advocates hardly connect them with quantum gravity.
} 
the philosophy of quantum theory. ${ }^{18}$ For our purposes, it suffices to note the four main connections of Everettian views with quantum gravity, specifically quantum cosmology.

The first connection has been evident from the earliest discussions of Everettian views. Namely, whatever the exact aims of a theory of quantum cosmology, in so far as it posits a 'quantum state of the universe', the Everettian promise to make sense of the quantum state of a closed system makes this interpretation particularly attractive.

The second connection concerns the more extreme Everettian view in which the universe is deemed literally to 'split'. In so far as this might involve some transformation of the topology of space, one naturally imagines implementing this with the aid of ideas from quantum gravity.

The third connection relates to decoherence, mentioned in question (c) above. Much recent work has shown decoherence to be a very efficient and ubiquitous mechanism for making interference terms small (and so for securing an apparent reduction of a quantum system's state vector); essentially by having the correlational information that these terms represent 'leak out' to the system's environment. Though this work in no way relies on Everettian views, Everettians can, and do, appeal to it in answering question (c). Furthermore, the work has been adapted to the discussion within quantum cosmology of how we 'see' a single classical space or spacetimes, despite the fact that in quantum cosmological models the quantum state of the universe assigns non-zero amplitude to many such spaces. The idea is that decoherence destroys the interference terms, 'hiding all but one'. (Typically, inhomogeneous modes of the gravitational field act as the environment of the homogeneous modes, which form the system.) For more discussion, see [5].

The fourth connection relates to time. Any Everettian view must specify not only probabilities for values of its preferred quantity at each time; it must also specify joint ('conjunctive') probabilities for values at sequences of times; i.e., a rule for the temporal evolution of these values. Traditionally, Everettians tended not to give such a rule; but recently they have done so, often in the context of the 'consistent-histories' approach to quantum theory.

There is a specific reason for quantum cosmologists to focus on the consistenthistories formalism, apart from the general need to specify a rule for the evolution of values. As we shall see in more detail in Section 3.3, quantum

\footnotetext{
${ }^{18}$ We do so, albeit briefly, in [1]; see also [3], and [4].
} 
gravity, and thereby quantum cosmology, is beset by 'the problem of time'. One response to this severe problem is to seek a new type of quantum theory in which time does not play the central role that it does in the standard approach. And precisely because the consistent-histories approach concerns many times, it suggests various ways in which the formalism of quantum theory can be generalised to be less dependent on the classical concept of time $[6,7]$.

This last point gives an example of an important, more general idea, which goes beyond the discussion of Everettian views. Namely, it is an example of how issues in quantum gravity can put pressure on the actual formalism of quantum theory - not just on some traditional interpretative views of it, such as the Copenhagen interpretation.

\subsubsection{Extra Values}

Again, we intend this approach to be a broad church. Like the Everettian views discussed above, it aims to interpret quantum theory - in particular, to solve the measurement problem - without invoking a collapse of the state vector. And it aims to do this by postulating values for some 'preferred quantity' or quantities, in addition to those given by the orthodox 'eigenvalueeigenstate link ${ }^{\prime 19}$; together with a rule for the evolution of such values.

But there are two differences from the Everettian views. First, 'Extra Values' makes no suggestion that there is a physically real 'branch' for every component in the resolution of the state vector in terms of the preferred quantity. (So there is no suggestion that 'branches splitting' prevents the detection of interference terms.) Second, 'Extra Values' aspires to be more precise from the outset about which quantity is preferred, and the dynamics of its values. ${ }^{20}$

\footnotetext{
${ }^{19}$ This asserts that a system has a real number $r$ as its value for a quantity $Q$ if and only if the quantum state is an eigenstate of $Q$ with eigenvalue $r$.

${ }^{20}$ Agreed, this second difference is a matter of degree. Furthermore, Everettians' imprecision about the preferred quantity, and the dynamics of its values, is partly just an accident, due to the facts that: (i) their view first developed within traditional quantum measurement theory, which invokes imprecise notions like 'apparatus' and 'pointerposition'; and (ii) they are willing to secure only the appearance of a definite macroscopic realm, not a truly definite one, and are therefore able to leave future psychophysics to specify the preferred quantities. But this matter of degree is no problem for us: our taxonomy of four approaches is not intended to be rigid.
} 
The best-known examples of this approach are the deBroglie-Bohm 'pilotwave' or 'causal' interpretation of quantum theory [8]; and the various kinds of modal interpretation [9]. Thus the pilot-wave interpretation of quantum mechanics postulates a definite value for the position of each point-particle, evolving according to a deterministic guidance equation. The corresponding interpretation of quantum field theory postulates a definite field configuration, and again a deterministic guidance equation. On the other hand, modal interpretations postulate that which quantity is 'preferred' depends on the state; and they consider various stochastic dynamics for values.

Within this approach, only the pilot-wave interpretation has been discussed in connection with quantum gravity. The main idea is to 'make a virtue of necessity', as follows. ${ }^{21}$ On the one hand, the guidance equations (at least as developed so far) require an absolute time structure, with respect to which the positions or field configurations evolve. (So for familiar quantum theories on Minkowski spacetime, the relativity of simultaneity, and the Lorentz-invariance of the theory, is lost at the fundamental level-but recovered at a phenomenological level.) On the other hand: in quantum gravity, one response to the problem of time is to 'blame' it on general relativity's allowing arbitrary foliations of spacetime; and then to postulate a preferred foliation of spacetime with respect to which quantum theory should be written. Most general relativists feel this response is too radical to countenance: they regard foliation-independence as an undeniable insight of relativity. But an advocate of the pilot-wave interpretation will reply that the virtues of that interpretation show that sacrificing fundamental Lorentz-invariance is a price worth paying in the context of flat spacetime; so why not also 'make a virtue of necessity' in the context of curved spacetime, i.e., general relativity?

Indeed, this suggestion has been developed in connection with one main approach to quantum gravity; namely, the quantum geometrodynamics version of the canonical quantum gravity programme. We will discuss this in more detail in Section 4.5. For the moment, we just note that the main idea of the pilot-wave interpretation of quantum geometrodynamics is to proceed by analogy with the interpretation of quantum field theories such as electrodynamics on flat spacetime. Specifically, a wave-function defined on 3-geometries (belonging to the 3-dimensional slices of a preferred foliation) evolves in time, and deterministically guides the evolution of a definite

\footnotetext{
${ }^{21}$ For more discussion, see the Chapter in this volume by Goldstein, and [8].
} 
3-geometry.

To sum up: 'Extra Values' preserves the usual unitary dynamics (the Schrödinger equation) of quantum theory, but adds equations describing the temporal evolution of its extra values. And the best developed version of 'Extra Values' - the pilot-wave interpretation - has been applied only to the quantum gravity programme based on quantum geometrodynamics.

\subsubsection{New Dynamics}

This approach is more radical than Extra Values. Instead of adding to the usual unitary dynamics of quantum theory, it replaces that dynamics; the motivation being to solve the measurement problem by dynamically suppressing the threatened superpositions of macroscopically distinguishable states. In the last fifteen years, there has been considerable development of this approach, especially in the wake of the 'spontaneous localization' theories of Ghirardi, Rimini and Weber [10], and Pearle [11].

This approach has natural links with quantum gravity. Indeed, from the point of view of physical theory itself, rather than its interpretation, it is a closer connection than those reviewed in the previous Subsections. For it is natural to suggest that the proposed deviation from the usual dynamics be induced by gravity (rather than being truly 'spontaneous'). This is natural for at least two reasons: (i) gravity is the only universal force we know, and hence the only force that can be guaranteed to be present in all physical interactions; and (ii) gravitational effects grow with the size of the objects concerned - and it is in the context of macroscopic objects that superpositions are particularly problematic.

We emphasise that this idea - that gravity is involved in the reduction of the state vector - is different from, and more radical than, the idea in Subsection 2.1.2 that some modes of the gravitational field might act as the environment of a system for a decoherence process that yields an apparent state-vector reduction. Here, there is no invocation of an environment; i.e., there is reduction for a strictly isolated system.

This idea has been pursued in various ways for several decades. In particular, adapting the idea to quantum gravity: since general relativity treats gravity as spacetime curvature, the most straightforward implementation of the idea will require that a quantum superposition of two spacetime geometries, corresponding to two macroscopically different distributions of mass- 
energy, should be suppressed after a very short time. Penrose has been particularly active in advocating this idea. More specific implementations of the idea involve variants of the spontaneous localization theories; for example, Pearle and Squires [12], which also contains a good bibliography. ${ }^{22}$

\subsection{Interpreting General Relativity}

We turn now to consider the conceptual problems of general relativity, especially those related to quantum gravity. However, our discussion will be briefer than that of Section 2.1, for the two reasons given at the start of the Section. First, general relativity is essentially a classical field theory, and its interpretation is less mysterious and controversial than that of quantum theory. Second, subsequent Sections will give ample discussion of the pressure that quantum gravity puts on general relativity.

Specifically, we shall confine ourselves to brief remarks about one aspect of the grand debate between 'absolute' versus 'relational' conceptions of space and time: namely, the question in what sense, if any, spacetime points are objects. ${ }^{23}$ (We shall discuss spacetime points, but most of the discussion could be straightforwardly rephrased as about whether regions in spacetime are objects; or, indeed - in a canonical approach - points or regions in 3-space, or in time.) For this question bears directly on the discussion in subsequent Sections of the treatment of spacetime in quantum gravity. ${ }^{24}$

The debate between 'absolute' and 'relational' conceptions of space and time has many strands. Nowadays, philosophers separate them, at least in part, by distinguishing various senses. For example, does a spatiotemporal structure being 'absolute' mean that it is 'non-dynamical', i.e., unaffected

\footnotetext{
${ }^{22}$ For further discussion and references, see the Chapter by Christian in this volume.

${ }^{23}$ We should stress that here we take the word 'object' in the 'post-Fregean' sense of anything that could be the referent of a singular term. In particular, it does not necessarily mean a 'thing out there' as a physicist might construe the phrase.

${ }^{24}$ Agreed, there are many other conceptual aspects of general relativity that bear on quantum gravity. We mentioned the philosophy of geometry at the end of Section 1.2. Another obvious example is the global structure of time, which bears on quantum cosmology. Here one faces such issues as: In what sense could a quantum event 'precede' the big-bang? This is related to the 'problem of time' in quantum gravity; which will be discussed later (Section 3.3); see also Part 2 of this volume, and Section 5 of our essay [1]). For a philosophical discussion of several other conceptual aspects of general relativity bearing on quantum gravity, see [13].
} 
by material events; or that it is not determined by (supervenient on) the spatiotemporal relations of material bodies? ${ }^{25}$ And what spatiotemporal structure does the 'absolutist' take to be absolute: space (as by Newton), or the 4-dimensional metric of spacetime, or the connection? Once these senses are distinguished, it becomes clear that general relativity supports 'relationism' in the senses that (i) its 4-dimensional metric and connection are dynamical; and (ii) in its generic models, no space, i.e., no foliation of spacetime, is preferred (whether dynamically or non-dynamically). On the other hand, it supports absolutism in the sense that the presence in the theory of the metric and connection is not determined by the spatiotemporal relations of material bodies.

But the consensus on these issues about relatively technical senses of 'absolute' leaves outstanding the question whether we should interpret general relativity as commited to the existence of spacetime points (or regions) as physical objects. We are wary of the 'Yes' answer to this question (which became popular in the 1960s, with the rise of scientific realism). But this is not just because we are wary of scientific realism; and in particular, of reifying the objects and structures postulated by physical theories (as discussed in Section 1.3). We also have two more specific reasons. The first contains a more general moral about reification; the second is specific to spacetime points.

(1): To explain the first reason, we should begin by admitting that it is especially tempting to take spacetime points as the fundamental physical objects of both our 'ingredient theories' - general relativity and quantum theory - and not just as points in mathematical models. There are two specific factors prompting this reification.

Roughly speaking, the first factor is this. As usually formulated, the theories agree with one another in postulating such points, endowed with the (highly sophisticated) structure of a differentiable manifold. But to be precise, one needs to respect the distinction between a (putative) physical spacetime point, and an (undeniably postulated!) point in a mathematical model of spacetime. So one should express this first factor by saying that, as usually formulated, the theories agree in postulating the latter points, i.e., those in the mathematical models. Even the most ardent realist must allow

\footnotetext{
${ }^{25}$ In any case, the rise of field theory undermines the contrast one naively learns in everyday life, between empty space and material bodies.
} 
this distinction in principle, if she is to avoid begging the question; though she may well go on to suggest that as a realist, she can take (and perhaps prefers to take) the physical points in which she believes, as elements of the mathematical model - say as the bottom-level elements in a set-theoretic definition of a manifold equipped with a Lorentzian metric and some matter fields.

The second factor is that, as usually formulated, the theories postulate the points initially, i.e., at the beginning of their formalism; the rest of physical reality being represented by mathematical structures (vector, tensor and operator fields etc.) defined over the points. ${ }^{26}$ Here, as in the first factor, to be precise - and to avoid begging the question - one must take these postulated points to be those in the mathematical models, not the putative physical points. The other structures representing fields etc. then become properties and relations among these postulated points; or, more generally, higher-order properties and relations; or in a formal formulation of the theory, set-theoretic surrogates for such properties and relations. And again, to avoid begging the question one must understand 'represent' as not committing one to the represented fields really being properties and relations. For that would commit one to there being objects which instantiated them; and these would no doubt be spacetime points and $n$-tuples of them.

However, notwithstanding these cautionary remarks, most people who bother to think about such matters succumb to Whitehead's fallacy of misplaced concreteness, by positing a one-to-one correspondence between what is undeniably real in the Platonic realm of mathematical form, and what is, more problematically, 'real' in the world of physical 'stuff'.

But tempting though this reification is, it is very questionable: not least because it overlooks the fact that these theories can be formulated in other (usually less well-known) ways, so as to postulate initial structures that, from the usual viewpoint, are complex structures defined on the points.

More precisely, the theories can be formulated so as to postulate initially not (i) mathematical objects that represent spacetime points (again, understanding 'represent' as not committing one to spacetime points being genuine physical objects); but rather (ii) mathematical objects that represent

\footnotetext{
${ }^{26}$ This of course reflects the rise, from the mid-nineteenth century onwards, of the fieldtheoretic conception of matter, whether classical or quantum. From another perspective, it reflects the dominant position of set theory in the foundations of mathematics.
} 
(again understood non-commitally) fields, and similar items - items that in the usual formulations are represented by complex mathematical structures (formally, set-theoretic constructions) defined over the initially-postulated representatives of spacetime points.

To give the flavour of such formulations, here is a standard example from the simpler setting of topological spaces, rather than differentiable manifolds. Consider a compact Hausdorff space $X$ and let $\mathcal{A}$ denote the ring of realvalued functions on $X$. Then it is a famous theorem in topology that both the set $X$ (i.e., its points) and the topology of $X$ can be uniquely reconstructed from just the algebraic structure of $\mathcal{A}$. Specifically, the closed subsets of $X$ are in one-to-one correspondence with the (closed) ideals in the commutative ring (actually, $C^{*}$-algebra) $\mathcal{A}$; and the points of $X$ correspond to maximal ideals in $\mathcal{A}$.

The implication of this result is that, from a mathematical perspective, a theory based on such a topological space - modeling, say, physical spacecan be formulated in such a way that the fundamental mathematical entity is not the set $X$ of spatial points - on which fields are then defined-but rather a commutative ring, on which spatial points are then defined: viz. as maximal ideals. Put in graphic terms, rather than writing $\phi(x)$, one writes $x(\phi)$ !

We emphasise that this idea is by no means esoteric from the perspective of theoretical physics. For example, the subject of 'non-commutative' geometry starts from precisely this situation and then posits a non-commutative extension of $\mathcal{A}$. In this case the algebra remains, but the points go in the sense that the algebra can no longer be written as an algebra of functions on anything.

(2): But there is also another reason for wariness about the existence of spacetime points as physical objects; a reason relating to symmetry transformations. The idea goes back to Leibniz; but in modern terms, it is as follows: given that a model of a theory represents a physical possibility, the model obtained by applying a global symmetry transformation to it describes the same physical possibility. In the context of spacetime theories, this idea means that taking points to be physical objects involves a distinction without a difference. So in particular, the existence of translation invariance in Newtonian or Minkowski spacetime shows that points should not be taken as physical objects. We think that most physicists would concur with this idea. 
In the context of general relativity, such considerations become perhaps yet more convincing, in view of Einstein's 'hole argument'. We cannot enter into details about this argument; which has interesting historical and physical, as well as philosophical, aspects. Suffice it to say that: (i) the argument applies not just to general relativity, but to any generally-covariant theory postulating a spacetime manifold; and (ii) according to the argument, general covariance (that is: the diffeomorphism-invariance of the theory), together with spacetime points being physical objects, implies a radical indeterminism: and such indeterminism is unacceptable - so that we should conclude that points are not physical objects. That is, the points occurring in the basesets of differentiable manifolds with which general relativity models spacetime should not be reified as physically real. ${ }^{27}$ We shall take up this theme again in more detail in Section 3.2.

\section{$3 \quad$ Introducing Quantum Gravity}

We turn now to our main project: surveying how quantum gravity suggests fundamental limitations in the familiar treatment of space and time that is common to the 'ingredient theories' - quantum theory and general relativity. In this Section, we first give some details about the variety of approaches to quantum gravity (Section 3.1). Then we give a more detailed discussion of two conceptual aspects relating specifically to spacetime: viz. the role of diffeomorphisms (Section 3.2) and the problem of time (Section 3.3). This will set the stage for the discussion in Section 4 of the treatment of spacetime in three of the main research programmes in quantum gravity.

\subsection{Approaches to Quantum Gravity}

In this Subsection, we begin to give a more detailed picture of quantum gravity research. We will first survey some motivations for studying quantum gravity (Section 3.1.1). Then we will consider, but reject, the view that

\footnotetext{
${ }^{27}$ This view of the argument seems to have been Einstein's own view, from the time of his discovery of general relativity in 1915 onwards. It was resuscitated for philosophers in [14]. For discussion and references (including replies on behalf of points being physically real) see [15].
} 
quantum gravity can be avoided (Section 3.1.2). Then we will describe four broad approaches to quantum gravity (Section 3.1.3).

\subsubsection{Motivations for Studying Quantum Gravity}

In surveying quantum gravity, it is useful to begin with the various motivations for studying the subject. For as we have seen, quantum gravity does not have a well-established body of 'facts' against which theories can be tested in the traditional way. In consequence, although some people's motivations refer to potential observations or experiments - particularly in the area of cosmology - most motivations are of a more internal nature: namely, the search for mathematical consistency, or the implementation of various quasi-philosophical views on the nature of space and time. And, since these different motivations have had a strong influence on researchers' technical approaches to the subject, it is important to appreciate them in order to understand what people have done in the past, and to be able to judge if they succeeded in their endeavours: since to be adjudged 'successful' a theory must presumably either point beyond itself to new or existing 'facts' in the world, or else achieve some of its own internal goals.

It is useful pedagogically to classify motivations for studying quantum gravity according to whether they pertain to the perspective of elementary particle physics and quantum field theory, or to the perspective of general relativity. As we shall see, this divide substantially affects one's approach to the subject, in terms of both the goals of research and the techniques employed.

\section{Motivations from the perspective of elementary particle physics and quantum field theory}

1. Matter is built from elementary particles that are described in quantum theoretical terms, and that certainly interact with each other gravitationally. Hence it is necessary to say something about the interface between quantum theory and general relativity, even if it is only to claim that, 'for all practical purposes', the subject can be ignored; (see Section 3.1.2 for more discussion).

2. Relativistic quantum field theory might only make proper sense if gravity is included from the outset. In particular, the short-distance diver- 
gences present in most such theories - including those that are renormalisable, but not truly finite - might be removed by a fundamental cut-off at the Planck energy. Superstring theory (see Section 4.4) is arguably the latest claimant to implement this idea.

3. A related claim is that considerations about quantum gravity will be a necessary ingredient in any fully consistent theory of the unification of the three non-gravitational forces of nature. ${ }^{28}$ The underlying idea here is as follows.

The mark of unification in a field theory is the equality of the coupling constants that determine the strengths of the different forces. However, in the quantum version these coupling 'constants' are energy dependent (they are said to 'run' with the energy) and therefore forces that are not unified at one energy may become so at a different one. It turns out that the running constants of the electromagnetic, weak and strong nuclear forces can be shown to 'meet', i.e., to be equal or at least approximately equal, at around $10^{20} \mathrm{Mev}$. The fact that $10^{20} \mathrm{Mev}$ is "quite close to" the Planck energy (viz. only two orders of magnitude less) then suggests that quantum gravity may have a role to play in this unification of forces.

This line of thought also prompts the more specific suggestion that a successful theory of quantum gravity must involve the unification of all four fundamental forces. As we shall see in Section 4, one of the key differences between the two most currently active research programmes, superstring theory and canonical quantum gravity, is that the former adopts this suggestion - it aims to to provide a scheme that encompasses all the forces - while the latter asserts that a quantum theory of pure gravity is possible.

\section{Motivations from the perspective of a general relativist}

1. Spacetime singularities arise inevitably in general relativity if the energy and momentum of any matter that is present satisfies certain,

\footnotetext{
${ }^{28}$ The four 'fundamental' forces recognised by present-day physicists are the electromagnetic force; the 'weak' nuclear force, that it responsible for radioactive decay; the 'strong' nuclear force, that binds together the constituents of nuclei; and the gravitational force.
} 
physically well-motivated, positivity conditions. It has long been hoped that the prediction of such pathological behaviour can be removed by the correct introduction of quantum effects.

2. A related point is that, once quantum mechanical effects are included, black holes produce Hawking radiation and, in the process, slowly lose their mass. But the nature of the final state of such a system is unclear, and much debated; providing another reason for studying quantum gravity.

3. Quantum gravity should play a vital role in the physics of the very early universe, not least because, in standard classical cosmology, the 'initial' event is an example of a spacetime singularity. Possible applications include:

(a) finding an explanation of why spacetime has a macroscopic dimension of four ${ }^{29}$

(b) accounting for the origin of the inflationary evolution that is believed by many cosmologists to describe the universe as it expanded after the initial big-bang.

4. Yet more ambitiously, one can hope that a theory of quantum gravity will provide a quantum cosmology, and thereby an understanding of the very origin of the universe in the big-bang as some type of quantum 'event'.

However, special problems are posed by quantum cosmology, for example about the interpretation of the quantum state (see Section 2.1); so one might well take the view that quantum gravity research should not get distracted by debating these problems. Certainly it would be a signal achievement to have a theory that successfully handled quantum theory and general relativity 'in the small', even if it could not be applied to the 'universe in its totality' - a problematic concept on any view! In any case, we shall from now on largely set aside quantum cosmology, and its special problems. ${ }^{30}$

\footnotetext{
${ }^{29}$ This does not exclude a Kaluza-Klein type higher dimension at Planckian scales. Indeed, superstring theory suggests strongly that something like this does occur.

${ }^{30}$ Our complementary essay [1], discusses this in some detail, especially as regards the
} 


\subsubsection{Can Quantum Gravity be Avoided?}

The argument is sometimes put forward that the Planck length $L_{P}:=$ $\left(G \hbar / c^{3}\right)^{\frac{1}{2}} \simeq 10^{-35} \mathrm{~m}$ is so small that there is no need to worry about quantum gravity except, perhaps, in recherché considerations of the extremely early universe -i.e., within a Planck time $\left(\simeq 10^{-42}\right.$ s) of the big-bang. However, as we hinted in the first motivation listed in Section 3.1.1:

- Such a claim is only really meaningful if a theory exists within whose framework genuine perturbative expansions in $L_{P} / L$ can be performed, where $L$ is the length scale at which the system is probed: one can then legitimately argue that quantum effects are ignorable if $L_{P} / L \ll 1$. So we must try to find a viable theory, even if we promptly declare it to be irrelevant for anything other than the physics of the very early universe.

- The argument concerning the size of $L_{P}$ neglects the possibility of nonperturbative effects - an idea that has often been associated with the claim that quantum gravity produces an intrinsic cut-off in quantum field theory.

A very different, and less radical, view is that - although we presumably need some sort of theory of quantum gravity for the types of reason listed in Section 3.1.1 - it is wrong to try to construct this theory by quantising the gravitational field, i.e., by applying a quantisation algorithm to general relativity (or to any other classical theory of gravity). We shall develop this distinction between the general idea of a theory of quantum gravity, and the more specific idea of quantised version of general relativity, immediately below (Section 3.1.3). For the moment, we mention some reasons advanced in support of this view.

- The metric tensor should not be viewed as a 'fundamental' field in physics, but rather as a phenomenological description of gravitational effects that applies only in regimes well away from those characterised by the Planck scale. Again, diverse reasons can be given for this viewpoint: we cite three. One example is superstring theory. Here,

Euclidean programme - which is mentioned in Section 4 below only as a species of canonical quantum gravity. 
the basic quantum entities are very different from those in classical general relativity, which is nevertheless recovered as a phenomenological description. Another - very different - example is Jacobson's rederivation of the Einstein field equations as an equation of state [16], which - presumably - it would be no more appropriate to 'quantise' than it would the equations of fluid dynamics. ${ }^{31}$ Yet a third example is Brown's view of the metric, even in special relativity, as phenomenological (see his Chapter in this volume).

- The gravitational field is concerned with the structure of space and time - and these are, par excellence, fundamentally classical in nature and mode of functioning. As we mentioned before, this might be defended from the viewpoint of (a version of) the Copenhagen interpretation (Section 2.1) - or even from a Kantian perspective (Section 1.3).

If it is indeed wrong to quantise the gravitational field (for whichever of the above reasons) it becomes an urgent question how matter-which presumably is subject to the laws of quantum theory - should be incorporated in the overall scheme. To discuss this, we shall focus on the so-called 'semiclassical quantum gravity' approach. Here, one replaces the right-hand-side of Einstein's field equations by a quantum expectation value, so as to couple a classical spacetime metric $\gamma$ to quantized matter by an equation of the form

$$
G_{\mu \nu}(\gamma)=\left\langle\psi\left|T_{\mu \nu}(g, \widehat{\phi})\right| \psi\right\rangle
$$

where $|\psi\rangle$ is some state in the Hilbert space of the quantised matter variables $\widehat{\phi}$. Thus the source for the gravitational field-i.e., the right hand side of Eq. (1) - is the expectation value of the energy-momentum tensor $T_{\mu \nu}$ of the quantised matter variables. In this context, we note the following:

- In the case of electromagnetism, the well-known analysis by Bohr and Rosenfeld [17] of the analogue of Eq. (1) concluded that the electromagnetic field had to be quantised to be consistent with the quantised nature of the matter to which it couples. However, the analogous argument for general relativity does not go through, and - in spite of much

\footnotetext{
${ }^{31}$ In 1971, one of us (CJI) took part in a public debate with John Stachel who challenged the former on this very issue. As a keen young quantum field theorist, CJI replied that he was delighted to quantise everything in sight. These days, not least because of the moderating influence of his philosopher friends, he is more cautious!
} 
discussion since then (for example, see Page and Geilker [19]) — there is arguably still no definitive proof that general relativity has to be quantised in some way.

- The right hand side of Eq. (1) generates a number of technical problems. For example, the expectation value has the familiar 'ultra-violet' divergences that come from the mathematically ill-defined short-distance behaviour of quantum fields. Regularisation methods only yield an unambiguous expression when the spacetime metric $\gamma$ is time-independent ${ }^{32}$ but there is no reason why a semi-classical metric should have this property. In addition, there have been many arguments implying that solutions to Eq. (1) are likely to be unstable against small perturbations, and-therefore - physically unacceptable.

- It is not at all clear how the state $|\psi\rangle$ is to be be chosen. In addition, if $\left|\psi_{1}\right\rangle$ and $\left|\psi_{2}\right\rangle$ are associated with a pair of solutions $\gamma_{1}$ and $\gamma_{2}$ to Eq. (1), there is no obvious connection between $\gamma_{1}$ and $\gamma_{2}$ and any solution associated with a linear combination of $\left|\psi_{1}\right\rangle$ and $\left|\psi_{2}\right\rangle$. Thus the quantum sector of the theory has curious non-linear features, and these generate many new problems of both a technical and a conceptual nature.

So much by way of reviewing the reasons one might give for avoiding quantum gravity. We make no claim that our 'replies' to these reasons - for example, our last two 'bullet-points' - are definitive. But we will from now on accept that some type of theory of quantum gravity should be sought.

\subsubsection{Four Types of Approach to Quantum Gravity}

In seeking such a theory, there are four broad types of approach one can adopt. We shall introduce them as answers to a series of questions; (questions which develop Section 1.1's contrast between the two strategies, quantisation and emergence). Broadly speaking, these questions will place them in an order of increasing radicalism. So, let us ask: should we adopt a diorthotic scheme in which general relativity is regarded as "just another classical field theory' to be quantised in a more-or-less standard way? Or should we instead

\footnotetext{
${ }^{32}$ More precisely, the spacetime metric has to be static or stationary.
} 
expect the theory of quantum gravity to look quite different from quantized general relativity, but nevertheless have general relativity emerge from it as some sort of low-energy (large-length) limit? This option itself breaks down into two alternatives, according to whether the theory is a quantization of some classical theory; or something that is constructed with no prior reference at all to a classical system. Or - a fourth alternative - should both quantum theory and general relativity emerge from a theory that looks quite different from both?

We will now develop the contrast between these four alternatives. Our survey in later Sections will not need to decide between them. But of the three programmes that we discuss in Section 4, two adopt the first alternative; and indeed, their implications for the treatment of spacetime are better understood than those of programmes adopting the other alternatives. Aspects of the third and fourth alternatives will be taken up in Section 5.

1. Quantise general relativity. The idea is to start with the classical theory of general relativity and then to apply some type of quantisation algorithm. This is intended to be analogous to the way in which the classical theory of, for example, an atom bound by the Coulomb potential is 'quantised' by replacing certain classical observables with self-adjoint operators on a Hilbert space; or, to take another example, the way in which classical electromagnetism is quantised to yield quantum electrodynamics. ${ }^{33}$

In the context of quantum gravity, the task is usually taken to be quantisation of the metric tensor regarded as a special type of field. In practice, the techniques that have been adopted fall into two classes: (i) those based on a spacetime approach to quantum field theory - in which the operator fields are defined on a four-dimensional manifold representing spacetime; and (ii) those based on a canonical approach - in which the operator fields are defined on a three-dimensional manifold representing physical space. We shall discuss (i) and (ii) in more detail in Sections 4.3 and 4.5 respectively.

2. General relativity as the low-energy limit of a quantization of a different classical theory. If a quantisation algorithm is applied to some classical theory, then that theory is naturally recovered as a classical limit of the ensuing

\footnotetext{
${ }^{33}$ Essentially this approach was also used in developing important elementary-particle physics theories, where there was no pre-existing classical theory; for example, the SalamGlashow-Weinberg electro-weak theory, and the quantum chromodynamics description of the strong nuclear force.
} 
quantum theory. In particular, this procedure provides a natural interpretation of the physical variables that arise in the quantum theory as the result of 'quantising' the corresponding classical theory.

But there are various senses of 'classical limit': it can refer to special states whose evolution over time follows classical laws, or to certain quantum quantities taking values in a range where classical theory is successful. So given a quantisation of a classical theory, some other classical theory might also be a classical limit of it, in some good sense.

Hence the idea in the context of quantum gravity, that general relativity might emerge as a low-energy (large-distance) classical limit of a quantum theory, that is given to us as a quantisation of a different classical theory. Of course, in view of our lack of data about quantum gravity, one expects it will be very hard to guess the correct classical theory from which to start.

But despite this difficulty, this type of approach is exemplified by the main current research programme: superstring theory, which quantizes a classical 'string theory' and yet has general relativity as a low-energy limit. We shall discuss this programme in Section 4.4. ${ }^{34}$ For the moment, suffice it to say that the dimensional nature of the basic Planck units lends support to the idea of a theory that could reproduce standard general relativity in regimes whose scales are well away from that of the Planck time, length, energy, etc. This remark is reinforced by a well-known body of work to the effect that, with appropriate caveats, general relativity is necessarily recovered as the low-energy limit of any interacting theory of massless spin-2 particles propagating on a Minkowski background, in which the energy and momentum are conserved [21]. The most notable example of this type is the theory of closed superstrings which has a natural massless, spin-2 excitation.

However, superstring theory is by no means the only example of this type of approach. For it is conservative, in that the classical 'string theory' that it quantises assumes the classical concept of a manifold. Roughly speaking, in perturbative superstring theory, the quantum variables are the functions that embed the string in a continuum spacetime manifold. But there have been more radical attempts to quantise aspects of space, or spacetime, itself. For example, there have been several attempts to construct a quantum theory of topology; and there have been attempts to quantize causal structures in which the underlying set is discrete. However, recovering general relativity

\footnotetext{
${ }^{34}$ For more discussion, see Part 4 of this volume.
} 
as a classical limit of theories of this type is by no means trivial since the implication is that the differentiable manifold structure of spacetime, not just its metric tensor, must be understood in some phenomenological sense. We will postpone to Section 5 discussion of these more radical attempts to quantise 'spacetime itself'.

Finally, a general point about this type of approach. Given the general scenario where we obtain a classical theory as a limit of a quantum theory, it is natural to wonder what would happen if one tried to quantise this derivative classical theory; (in the case of interest to us, general relativity). Generally speaking, this does not give back the initial quantum theory. That is unsurprising, in view of our comments above about the variety of classical limits. But we should also make two more specific remarks. First, one reason why one does not get back the initial quantum theory may be that the classical limit is non-renormalisable: this is well known to be the case for general relativity (as we will discuss in Section 4.3). But second: this feature does not render the 're-quantisation' procedure completely useless. Indeed, genuine quantum predictions can be obtained by empirically fixing the appropriate number of renormalisation constants, where what is 'appropriate' is determined by the energy at which the theory is to be employed. Theories of this type are called "effective field theories" and are a valuable tool in modern theoretical physics. For a recent review in the context of general relativity see [22].

3. General relativity as the low-energy limit of a quantum theory that is not a quantization of a classical theory. The procedure of going from classical to quantum has become so ubiquitous (for example, look at the content of a typical undergraduate lecture course on quantum theory!) that one might be tempted to assume that all quantum theories necessarily arise in this way. However, there is no good reason why this should be so. So it is certainly reasonable to consider the construction of a quantum theory ab initio with no fundamental reference to an underlying classical theory-for example as a representation of some group or algebra. The question then arises whether a quantum theory of this type may have a classical limit of some sort, even though it is not obtained by the quantisation of such. A good example of a quantum theory of this kind was the 'current algebra' approach to stronginteraction physics that was intensely studied in the 1960s.

Of course, one might well fear that in quantum gravity, with its dire lack of 
data, this type of approach will be at least as hard to implement successfully as is the previous one: the correct group or algebra might be as hard to guess as is the correct classical system to quantise in the previous approach. However, recent developments in understanding the non-perturbative aspects of superstring theory suggest that this type of approach may well come to the fore in that programme; (see Section 4.4).

4. Start ab initio with a radically new theory. The idea here is that both classical general relativity and standard quantum theory emerge from a theory that looks very different from both. Such a theory would indeed be radically new. For recall that we classified as examples of the second type of approach above, quantisations of spatial or spatiotemporal structure other than the metric; for example, quantisations of topology or causal structure. So the kind of theory envisaged here would somehow be still more radical than that; presumably by not being a quantum theory, even in a broad sense-for example, in the sense of states giving amplitudes to the values of quantities, whose norms squared give probabilities.

Of course, very little is known about potential schemes of this type; let alone whether it is necessary to adopt such an iconoclastic position in order to solve the problem of quantum gravity. We shall mention some possible clues in Section 5. For the moment, we want just to emphasise the philosophical interest of this type of approach. For it is often motivated by the view that the basic ideas behind general relativity and quantum theory are so fundamentally incompatible that any complete reconciliation will necessitate a total rethinking of the central categories of space, time and matter. And as we mentioned in Section 1.2 (item 3), we like to think that philosophy could have a role in that enterprise!

As mentioned above, all four types of approach have been followed in the past (albeit in a very limited way in regard to the third and fourth types). Until fifteen years ago, the bulk of the effort was devoted to the first - the active quantisation of classical general relativity; so that two of the three programmes reviewed in Section 4 are of this type. But nowadays the dominant programme, viz. superstrings, is of the second type; although the second most dominant programme - canonical quantum gravity in the Ashtekar approach - is of the first type; and both these programmes have touches of the third type. In short, it remains a matter of vigorous debate which of these types of approach will ultimately prove to be the most fruitful. 


\subsection{The Role of Diffeomorphisms}

To set the stage for Section 4, we devote the rest of this Section to discussing two conceptual aspects that relate specifically to spacetime: viz. the role of diffeomorphisms (this Subsection); and the problem of time (Section 3.3).

\subsubsection{Spacetime Diffeomorphisms in Classical General Relativity}

The group of spacetime diffeomorphisms $\mathcal{D}$ plays a key role in classical general relativity; and its status in quantum gravity raises some major conceptual issues. $^{35}$

In considering these matters, it is important to distinguish between the pseudo-group of local coordinate transformations and the genuine group $\mathcal{D}$ of global diffeomorphisms. Compatibility with the former can be taken to imply that the theory should be written using tensorial objects on spacetime. On the other hand, diffeomorphisms are active transformations of spacetime, and invariance under $\mathcal{D}$ implies, we take it, that the points in spacetime have no direct physical significance (see the discussion of realism, and the hole argument in Sections 1.3, and 2.2.) Of course, this is also true in special relativity but it is mitigated there by the existence of inertial reference frames that can be transformed into each other by the Poincaré group of isometries of the Minkowski metric.

Put somewhat differently, the action of $\mathcal{D}$ induces an action on the space of spacetime fields, and the only thing that has immediate physical meaning is the space of equivalence classes under this action: i.e., two field configurations are regarded as physically equivalent if they are connected by a diffeomorphism transformation. Technically, this is analogous in certain respects to the situation in electromagnetism whereby a vector potential $A_{\mu}$ is equivalent to $A_{\mu}+\partial_{\mu} f$ for all functions $f$. However, there is an important difference between electromagnetism and general relativity. Electromagnetic gauge transformations occur at a fixed spacetime point $X$, and the physical configurations can be identified with the values of the field tensor $F_{\mu \nu}(X)$,

\footnotetext{
${ }^{35}$ The diffeomorphisms concerned are those of compact support, i.e., they are equal to the unit map outside some closed and bounded region of the spacetime (for some purposes, more subtle 'fall-off' rates at infinity may be appropriate). Thus, for example, a Poincarégroup transformation of Minkowski spacetime is not included. This restriction is imposed because the role of transformations with a non-trivial action in the asymptotic regions of spacetime is quite different from those that act trivially.
} 
which depends locally on points of $\mathcal{M}$. On the other hand, a diffeomorphism maps one spacetime point into another, and therefore the obvious way of constructing a diffeomorphism-invariant object is to take a scalar function of spacetime fields and integrate it over the whole of spacetime, which gives something that is very non-local. The idea that 'physical observables' are naturally non-local is an important ingredient in some approaches to quantum gravity.

\subsubsection{Diffeomorphisms in Quantum Gravity}

The role of diffeomorphisms in quantum gravity depends strongly on the approach taken to the subject. For example, if the structure of classical relativity is expected to appear only in a low-energy limit - as, for example, is the case for superstring theory - there is no strong reason to suppose that the group of spacetime diffeomorphisms $\mathcal{D}$ will play any fundamental role in the quantum theory. On the other hand, in schemes which involve the active quantisation of the classical gravitational field, $\mathcal{D}$ is likely to be a key ingredient in forcing the quantum theory to comply with the demands of general relativity. However, it should be noted that the situation in 'canonical' quantum gravity is less clear-cut: this programme is based on a prior decomposition into space plus time, and this is bound to obscure the role of spacetime diffeomorphisms.

In general terms, there are at least three ${ }^{36}$ ways in which $\mathcal{D}$ could appear in the quantum theory; which will be exemplified in the programmes surveyed in Sections 4 et seq.:

(i) as an exact covariance/invariance group; ${ }^{37}$

(ii) as a subgroup of a bigger group;

\footnotetext{
${ }^{36}$ Another possibility - not exemplified in the programmes surveyed in this paper - is that the diffeomorphism group $\mathcal{D}$ could be related to a bigger group $G$ in a projective way: i.e., there is some normal subgroup $K$ of $G$ so that $G / K \simeq \mathcal{D}$.

${ }^{37}$ Here we should distinguish the invariance of each individual expression in a theory's formalism from the invariance of all the physically measurable values of quantities; the latter being of course a weaker property. In fact, most of the programmes whose basic framework treats $\mathcal{D}$ as an exact covariance group in practice 'fix a gauge' for their formalism, and so work with non-covariant/non-diffeomorphism-invariant expressions, and then show physically measurable values to be gauge-invariant. So in practice, these programmes enjoy only the weaker property.
} 
(iii) as a limited concept associated with a phenomenological view of spacetime (or space).

In the first two options one could say that the diffeomorphisms form a 'precise' concept since the mathematical object that occurs in the formalism is exactly the classical group $\mathcal{D}$. The third option, (iii), is somewhat different and flows naturally from the view that spacetime is a phenomenological concept of limited applicability: the same would then be expected for the diffeomorphisms of the manifold that models spacetime in this limited sense. We shall say more about this in Section 5 .

The idea that $\mathcal{D}$ is an exact covariance/invariance group (option (i) above) plays a key role in several approaches to quantum gravity. For example, (i) is one of the central properties of so-called 'topological quantum field theory', which seems to have potential applications in quantum gravity. And we will see in Section 4 that (i) also plays a major role in the particle-physics programme (Section 4.3), albeit with the qualification mentioned in footnote 37 above [to option (i)]; and in a less clear-cut way, in canonical quantum gravity.

On the other hand, the idea that $\mathcal{D}$ is a subgroup of a bigger covariance group (option (ii) above) is endorsed by the perturbative approach to superstring theory. In short, the idea is that the extra fields associated with supersymmetry lead to a much larger covariance group; more details in Section 4.4.

\subsection{The Problem of Time}

Closely related to the role of diffeomorphisms is the infamous 'problem of time'. This problem is central in any approach to quantum gravity that assigns a significant prima facie role to classical general relativity (unlike, say, superstring theory). For the problem arises from the very different roles played by the concept of time in quantum theory and in general relativity; and the problem lies at the heart of many of the deepest conceptual issues in such approaches to quantum gravity. To present the problem, we will consider the roles of time, first in quantum theory, and then in general relativity. ${ }^{38}$

\footnotetext{
${ }^{38}$ For much fuller expositions of the problem from the point of view of canonical quantum gravity, see the Chapters by Belot \& Earman, and Weinstein, in Part 2 of this volume; see also [23], [24].
} 


\subsubsection{Time in Quantum Theory}

In quantum theory, time is not a physical quantity in the normal sense, since it is not represented by an operator. Rather, it is treated as a background parameter which, as in classical physics, is used to mark the evolution of the system; witness the parameter $t$ in the time-dependent Schrödinger equation. ${ }^{39}$

Besides, the idea of an event happening at a given time plays a crucial role in the technical and conceptual foundations of quantum theory:

- One of the central requirements of the scalar product on the Hilbert space of states is that it is conserved under the time evolution given by the Schrödinger equation. This is closely connected to the unitarity requirement that probabilities always sum to one.

- More generally, a key ingredient in the construction of the Hilbert space for a quantum system is the selection of a complete set of quantities that are required to commute at a fixed value of time.

- Conceptually, the notion of measuring a quantity at a given time, to find its value at that time, is a fundamental ingredient of both the minimal statistical interpretation of the theory, and the Copenhagen interpretation (see Section 2.1.1).

Furthermore, all these ideas can be extended to systems that are compatible with special relativity: the unique time system of Newtonian physics is simply replaced with the set of relativistic inertial reference frames. The quantum theory can be made independent of a choice of frame, provided that the theory carries a unitary representation of the Poincaré group of isometries of the metric of Minkowski spacetime. In the case of a relativistic quantum field theory, the existence of such a representation is closely related to the microcausality requirement that fields evaluated at spacelike-separated points must commute. For example, a scalar quantum field $\widehat{\phi}(X)$ satisfies the commutation relations

$$
[\widehat{\phi}(X), \widehat{\phi}(Y)]=0
$$

\footnotetext{
${ }^{39}$ This is why the meaning assigned to the time-energy uncertainty relation $\delta t \delta E \geq \frac{1}{2} \hbar$ is quite different from that associated with, for example, the position and the momentum of a particle.
} 
whenever the spacetime points $X$ and $Y$ are spacelike separated.

Finally, we note that this background time is truly an abstraction in the sense that according to quantum theory, no physical clock can provide a precise measure of it [25]: there is always a small probability that a real clock will sometimes run backwards with respect to it.

\subsubsection{Time in General Relativity; and the Problem of Time}

When we turn to classical general relativity, the treatment of time is very different. Time is not treated as a background parameter, even in the liberal sense used in special relativity, viz. as an aspect of a fixed, background spacetime structure. Rather, what counts as a choice of a time (i.e of a timelike direction) is influenced by what matter is present; (as is, of course, the spatial metrical structure). The existence of many such times is reflected in the fact that if the spacetime manifold has a topology that enables it to be foliated as a one-parameter family of spacelike surfaces, this can generally be done in many ways - without any subset of foliations being singled out in the way families of inertial reference frames are singled out in special relativity. From one perspective, each such parameter might be regarded as a legitimate definition of (global) time. However, in general, there is no way of selecting a particular foliation, or a special family of such, that is 'natural' within the context of the theory alone. In particular, these definitions of time are in general unphysical, in that they provide no hint as to how their time might be measured or registered.

But the main problem about time in general relativity arises when we turn to quantum gravity, where the disparate nature of the treatments of time in quantum theory and in general relativity becomes of paramount significance. We shall see various more specific versions of this problem in each of the research programmes reviewed in Section 4. But for the moment, we introduce the problem in general terms.

General relativity accustoms us to the ideas that: (i) the causal structure of spacetime depends on the metric structure, represented by the metric tensor $\gamma$; and (ii) the metric and causal structures are influenced by matter, and so vary from one model of the theory to another. In general relativity, these ideas are 'kept under control' in the sense that in each model, there is of course a single metric tensor $\gamma$, representing a single metric and causal structure. But once we embark on constructing a quantum theory of gravity, 
we expect some sort of quantum fluctuations in the metric, and so also in the causal structure. But in that case, how are we to formulate a quantum theory with a fluctuating causal structure?

This general statement of the problem is clearly relevant if one proposes a spacetime-oriented approach to formulating the quantum theory; since then one's prototype quantum theories will emphasise a fixed background causal structure. But the same statement of the problem arises on various other approaches to quantum gravity. For example, if one takes the view that the spacetime metric is only a coarse-grained, phenomenological construct of some type, then so is the causal structure. And again, the question arises how we are to formulate a quantum theory with such a causal structure. ${ }^{40}$

Though this problem is at bottom conceptual, it has clear technical aspects. In particular, a probabilistic causal structure poses severe technical problems for relativistic quantum field theory, whose standard formulation presupposes a fixed causal structure. For example, a quantum scalar field satisfies the microcausal commutation relations in Eq. (2), whereby fields evaluated at spacelike separated spacetime points commute. However, the concept of two points being spacelike separated has no meaning if the spacetime metric is probabilistic or phenomenological. In the former case, the most likely scenario is that the right hand side of the commutator in Eq. (2) never vanishes, thereby removing one of the foundations of conventional quantum field theory.

In practice, the techniques that have been used to address the problem of time fall into one of the following three strategies, to all of which we shall return in Section 4:

1. Use a fixed background metric - often chosen to be that of Minkowski spacetime - to define a fiducial causal structure with respect to which standard quantum field theoretical techniques can be employed. This is the strategy adopted by the old particle-physics programme, using Minkowski spacetime (Section 4.3).

This strategy raises questions about how the background structure is to be chosen. Of course, Minkowski spacetime seems very natural from

\footnotetext{
${ }^{40}$ Note that this general statement of the problem of time would have an analogue in a stochastic version of classical general relativity, in which the metric tensor is regarded as a random variable. Of course, the other difficult problem of understanding what is meant by 'superpositions' of spacetime geometries would be absent in this case.
} 
the perspective of standard quantum field theory, but it is rather arbitrary when seen in the context of general relativity. One possibility is that the background structure could come from a contingent feature of the actual universe; for example, the $3^{0} \mathrm{~K}$ microwave background radiation. However, structure of this type is approximate and is therefore applicable only if fine details are ignored. In addition, the problem of rigorously constructing (even free) quantum fields has only been solved for a very small number of background spacetimes; certainly there is no reason to suppose that well-defined quantum field theories exist on a generic spacetime manifold. Also, there is a general matter of principle: should we require a theory of quantum gravity to work for 'all possible' universes (however that is made precise), or can it depend on special features of the actual one in which we live?

2. Accept the fact that there is no background spacetime reference system and attempt to locate events, both spatially and temporally, with specific functionals of the gravitational and other fields. This important idea is of course motivated by the analysis of the 'hole argument' and spacetime diffeomorphisms (see Sections 2.2 and 3.2). Thus the idea is that for the example of a scalar field $\phi$, the value $\phi(X)$ of $\phi$ at a particular spacetime point $X$ has no physical meaning because of the action of the spacetime diffeomorphism group; but, the value of $\phi$ where something 'is' does have a physical meaning in the sense that ' $\phi$ (thing)' is diffeomorphism invariant. In practice, this strategy seems only to have been adopted by some of the approaches to the problem of time as it manifests itself in canonical quantum gravity.

3. And indeed, one approach is to drop spacetime methods and instead adopt a canonical approach to general relativity, so that the basic ingredients are geometrical fields on a three-dimensional manifold. The problem then is to reconstruct some type of spatio-temporal picture within which the quantum calculations can be interpreted. This is the strategy adopted by the canonical quantum gravity programme (Section 4.5).

Studies of the problem of time in canonical quantum gravity raise the alluring question whether a meaningful quantum theory can be constructed in a way that contains no fundamental reference to time at 
all. That this is a far from trivial matter is shown by our earlier remarks about the crucial role of time in conventional quantum theory (see the references in footnote 38 ).

\section{Research Programmes in Quantum Grav- ity}

As we have seen, we are far from having an 'axiomatic' framework for quantum gravity, or even a broad consensus about what to strive for beyond the minimal requirement that the theory should reproduce classical general relativity and normal quantum theory in the appropriate domains - usually taken to be all physical regimes well away from those characterised by the Planck length.

In this Section, we shall focus on three specific research programmes. Our aim is not to review the technical status of these programmes, but rather to explore their treatments of spacetime. Of these three programmes, two are the main current focus for work in quantum gravity: superstring theory, and canonical quantum gravity (in the version called 'the Ashtekar programme'). These programmes complement each other nicely, and enable the special ideas of either of them to be viewed in a different perspective by invoking the other - a feature that is rather useful in a subject that lacks unequivocal experimental data! For example, they exemplify the choice of approaches we discussed in Section 3.1.3, about whether to quantise general relativity, or to have classical general relativity emerge from a quantum theory of something quite different. Superstring theory takes the latter approach; canonical quantum gravity the former.

The other programme we shall discuss is a spacetime-oriented quantization of general relativity, which we dub 'the particle-physics programme'. This programme is no longer regarded as capable of providing a full theory of quantum gravity; but it predated, and so influenced, both the other two programmes, and this means that discussing its own treatment of spacetime will form a helpful backdrop to discussing theirs. ${ }^{41}$

\footnotetext{
${ }^{41}$ Clearly, one could also discuss other programmes so as to provide still more of a backdrop to the two main ones. One obvious choice is the Euclidean programme, which can be viewed as a spacetime-oriented species of the traditional (geometrodynamic, rather
} 
All three programmes postulate at the fundamental level a spacetime manifold. But it may differ in its dimension, metric structure etc. from the 4-dimensional Lorentzian manifold familiar from classical general relativity. And, in fact, these programmes suggest limitations to the applicability of the concept of a spacetime manifold itself. We shall explore this possibility further in Section 5.

We begin in Section 4.1 by listing four topics that will act as 'probes' in our survey of how concepts of space and time are treated in these programmes. This is followed by some historical orientation to these programmes (Section 4.2), and then we consider them seriatim, in the following order: the particle-physics programme (Section 4.3); supergravity and superstrings (Section 4.4); and canonical quantum gravity (Section 4.5).

\subsection{Focussing the Question: How is Spacetime Treated?}

We will take the following four topics as 'probes' in our survey of how the concept of spacetime is treated in the various quantum gravity programmes. We present them as a sequence of questions; but of course they overlap with one another.

1. Use of standard quantum theory. Are the technical formalism and conceptual framework of present-day quantum theory adequate for the programme's envisaged theory of quantum gravity? In particular, do any features of the programme suggest advantages, or indeed disadvantages, of the 'heterodox' interpretations of quantum theory, discussed in Sections 2.1.2-2.1.4?

2. Use of standard spacetime concepts. How much of the familiar treatment of spatio-temporal concepts, adopted by general relativity and quantum theory, does the programme adopt? In particular, does it model spacetime as a 4-dimensional differentiable manifold? If so, does it add to this manifold a quantized metric tensor? And if so, what exactly is the relation of this to a classical Lorentzian metric on the manifold?

than Ashtekar) version of canonical quantum gravity. But we ignore it here, since (i) it is especially connected with quantum cosmology, which we have set aside; and (ii) we discuss it in [1]. 
3. The spacetime diffeomorphism group. Assuming the programme models spacetime as a manifold, what role does it assign to the group of spacetime diffeomorphisms? Or if it decomposes spacetime into space and time, what role is given to spatial diffeomorphisms?

4. The problem of time. How does the problem of time manifest itself in the programme, and how does the programme address it? In particular: how much of the familiar treatment of spacetime must be retained for the envisaged theory of quantum gravity to be constructed? Must both metric and manifold be fixed; or can we work with a fixed background manifold, but no background metric? Could we also do without the background manifold?

\subsection{Some Historical Background to the Three Pro- grammes}

To introduce the survey of our three chosen research programmes, it is useful to sketch some of the historical development of quantum gravity research - a development from which these programmes have all sprung.

The early history of attempts to quantise general relativity goes back at least to the 1960s and was marked by a deep division of opinion about whether quantum gravity should be tackled from a spacetime perspective- the socalled 'covariant' approach, whose leading champion was Bryce DeWitt - or from a 'canonical' approach, in which spacetime is decomposed into space plus time before the theory is quantised.

The early predominance of the canonical programme stemmed partly from the fact that the attitude in the 1960s towards quantum field theory was very different from that of today. With the exception of quantum electrodynamics, quantum field theory was poorly rated as a fundamental way of describing the interactions of elementary particles. Instead, this was the era of the S-matrix, the Chew axioms, Regge poles, and - towards the end of that period - the dual resonance model and the Veneziano amplitude that led eventually to superstring theory.

In so far as it was invoked at all in strong interaction physics, quantum field theory was mainly used as a phenomenological tool to explore the predictions of current algebra, which was thought to be more fundamental. When quantum field theory was studied seriously, it was largely in the context of 
an 'axiomatic' programme - such as the Wightman axioms for the $n$-point functions.

This neglect of quantum field theory influenced the way quantum gravity developed. In particular - with a few notable exceptions - physicists trained in particle physics and quantum field theory were not interested in quantum gravity, and the subject was mainly left to those whose primary training had been in general relativity. This imparted a special flavour to much of the work in that era. In particular, the geometrical aspects of the theory were often emphasised at the expense of quantum field theoretic issues - thereby giving rise to a tension that has affected the subject to this day.

However, a major change took place in the early 1970s when t'Hooft demonstrated the renormalisability of quantised Yang-Mills theory. Although not directly connected with gravity, these results had a strong effect on attitudes towards quantum field theory in general, and reawakened a wide interest in the subject. One spin-off was that many young workers in particle physics became intrigued by the challenge of applying the new methods to quantum gravity. This led to a revival of the covariant approach; more specifically, to the particle-physics programme (Section 4.3), and thereby eventually to supergravity and superstring theory (Section 4.4).

On the other hand, the canonical programme has also continued to flourish since the 1970s; indeed, until the relatively recent advent of 'superstring cosmology', canonical quantum gravity provided the only technical framework in which to discuss quantum cosmology. A major development in canonical quantum gravity was Ashtekar's discovery in 1986 of a new set of variables that dramatically simplifies the intractable Wheeler-DeWitt equation which lies at the heart of the programme's quantum formalism.

\subsection{The Particle-Physics Programme}

\subsubsection{The basic ideas}

In this programme, the basic entity is the graviton - the quantum of the gravitational field. Such a particle is deemed to propagate in a background Minkowski spacetime, and-like all elementary particles - is associated with a specific representation of the Poincare group which is labelled by its mass and its spin. The possible values of mass and spin are sharply limited by the physical functions which the graviton is to serve. In particular, replication of 
the inverse-square law behaviour of the static gravitational force requires the graviton to have mass zero, and the spin must be either 0 or $2 \hbar$. However, zero spin is associated with a scalar field $\phi(X)$, whereas spin-two comes from a symmetric Lorentz tensor field $h_{\mu \nu}(X)$; the obvious implication is that these spin values correspond to Newtonian gravity and general relativity respectively.

The key to relating spin-2 particles and general relativity is to fix the background topology and differential structure of spacetime $\mathcal{M}$ to be that of Minkowski spacetime, and then to write the Lorentzian metric $\gamma$ on $\mathcal{M}$ as

$$
\gamma_{\alpha \beta}(X)=\eta_{\alpha \beta}+\kappa h_{\alpha \beta}(X)
$$

Here, $h$ measures the departure of $\gamma$ from the flat spacetime metric $\eta$ and is regarded as the 'physical' gravitational field with the coupling constant $\kappa^{2}=8 \pi G / c^{2}$ where $G$ is Newton's constant.

The use of the expansion in Eq. (3) strongly suggests a perturbative approach in which quantum gravity is seen as a theory of small quantum fluctuations around a background Minkowski spacetime. Indeed, when this expansion is substituted into the Einstein-Hilbert action for pure gravity, $S=\int d^{4} X|\gamma|^{\frac{1}{2}} R(\gamma)$ (where $R(\gamma)$ is the scalar curvature), it yields (i) a term that is bilinear in the fields $h$ and which - when quantised in a standard way-gives a theory of non-interacting, massless spin-2 gravitons; and (ii) a series of higher-order terms that describe the interactions of the gravitons with each other. Thus a typical task would be to compute the probabilities for various numbers of gravitons to scatter with each other and with the quanta of whatever matter fields might be added to the system.

This approach to quantum gravity has some problematic conceptual features (see below). But, nonetheless, had it worked it would have been a major result, and would undoubtedly have triggered a substantial effort to construct a spacetime-focussed quantum gravity theory in a non-perturbative way. A good analogue is the great increase in studies of lattice gauge theory that followed the proof by t'Hooft that Yang-Mills theory is perturbatively renormalisable.

However, this is not what happened. Instead, a number of calculations were performed around 1973 that confirmed earlier suspicions that perturbative quantum gravity is non-renormalisable ${ }^{42}$. There have been four main

\footnotetext{
${ }^{42}$ Full references can be found in reviews written around that time; for example, in the
} 
reactions to this situation:

- Adopt the view in which general relativity is an "effective field theory" and simply add as many empirically determined counter-terms as are appropriate at the energy concerned. The ensuing structure will break down at the Planck scale but a pragmatic particle physicist might argue that this is of no importance since the Planck energy is so much larger than anything that could be feasibly attainable in any foreseeable particle accelerator (see the discussion at the end of the second approach in Section 3.1.3.)

- Continue to use standard perturbative quantum field theory but change the classical theory of general relativity so that the quantum theory becomes renormalisable. Examples of such attempts include (i) adding higher powers of the Riemann curvature $R_{\beta \mu \nu}^{\alpha}(\gamma)$ to the action; and (ii) supergravity (see Section 4.4 below).

- Keep classical general relativity as it is, but develop quantisation methods that are intrinsically non-perturbative. Examples of this philosophy are 'Regge calculus' (which involves simplicial approximations to spacetime) and techniques based on lattice gauge theory. Of particular importance in recent years is the Ashtekar programme for canonical quantisation which is fundamentally non-perturbative (see Section 4.5.2 below).

- Adopt the view that the non-renormalisability of perturbative quantum gravity is a catastrophic failure that requires a very different type of approach. In terms of the classification in Section 3.1.3, this would mean adopting its second, or third or fourth types of approach: quantising a classical theory that is quite different from general relativity (such as a string theory); or having general relativity emerge as a low-energy limit of a quantum theory that is not a quantisation of any classical system; or having it and quantum theory both emerge from something completely different.

proceedings of the first two Oxford conferences on quantum gravity [26, 27]. 


\subsubsection{Spacetime according to the Particle-Physics Programme}

The response given by this programme to our four conceptual probes presented in Section 4.1 is as follows.

1. Use of standard quantum theory. The basic technical ideas of standard quantum theory are employed, suitably adapted to handle the gauge structure of the theory of massless spin-2 particles. Furthermore, the traditional, Copenhagen interpretation of the theory is applicable (even if not right!), in that the background Minkowski metric and spacetime manifold are available to serve as the classical framework, in which measurements of the quantum system, according to this interpretation, are to be made.

Of course, other interpretations of quantum theory discussed in Section 2.1, such as Everettian or pilot-wave interpretations, may well also be applicable to this programme. Our present point is simply that the particle-physics programme gives no special reasons in favour of such interpretations.

2. Use of standard spacetime concepts. The background manifold and metric are described in the language of standard differential geometry. Note that, from a physical perspective, the restriction to a specific background topology means a scheme of this type is not well adapted to addressing some of the most interesting questions in quantum gravity such as black-hole phenomena, quantum cosmology, the idea of possible spacetime 'phase changes' etc.

3. The spacetime diffeomorphism group. The action of the group of spacetime diffeomorphisms is usually studied infinitesimally. The transformation of the graviton field $h_{\mu \nu}(X)$ under a vector-field generator $\xi$ of such a diffeomorphism is simply $h_{\mu \nu}(X) \mapsto h_{\mu \nu}(X)+\partial_{\mu} \xi_{\nu}(X)+\partial_{\nu} \xi_{\mu}(X)$, which is very reminiscent of the gauge transformations of the electromagnetic vector potential, $A_{\mu}(X) \mapsto A_{\mu}(X)+\partial_{\mu} \phi(X)$.

Indeed, in this infinitesimal sense, the effect of spacetime diffeomorphisms is strictly analogous to the conventional gauge transformations of electromagnetism or Yang-Mills theory (in that spacetime points are fixed); and the same type of quantisation procedure can be used. In particular, the invariance of the quantum theory under these transformations is reflected in a set of 'Ward identities' that must be satisfied by the vacuum expectation values of time-ordered products (the ' $n$-point functions') of the operator field at different spacetime points.

4. The problem of time The background metric $\eta$ provides a fixed causal 
structure with the associated family of Lorentzian inertial frames. Thus, at this level, there is no problem of time. The causal structure also allows a notion of microcausality, thereby permitting a conventional type of relativistic quantum field theory to be applied to the field $h_{\alpha \beta}$.

However, many people object strongly to an expansion like Eq. (3) since it is unclear how this background causal structure is to be related to the physical one; or, indeed, what the latter really means. For example, does the 'physical' causal structure depend on the state of the quantum system? There have certainly been conjectures to the effect that a non-perturbative quantisation of this system would lead to quantum fluctuations of the causal structure around a quantum-averaged background that is not the original Minkowskian metric. As emphasised earlier, it is not clear what happens to the microcausal commutativity condition in such circumstances; or, indeed, what is meant in general by 'causality' and 'time' in a system whose lightcones are themselves the subject of quantum fluctuations.

\subsection{The Superstrings Programme}

\subsubsection{The introduction of supersymmetry}

When confronted with the non-renormalisability of covariant quantum gravity, the majority of particle physicists followed a line motivated by the successful transition from the old non-renormalisable theory of the weak interactions (the 'four-fermion' theory) to the new renormalisable unification of the weak and electromagnetic forces found by Salam, Glashow and Weinberg. Thus the aim was to construct a well-defined theory of quantum gravity by adding carefully chosen matter fields to the classical theory of general relativity with the hope that the ultraviolet divergences would cancel, leaving a theory that is perturbatively well-behaved.

A key observation in this respect is that the divergence associated with a loop of gravitons might possibly be cancelled by introducing fermions, on the grounds that the numerical sign of a loop of virtual fermions is opposite to that of a loop of bosons. With this motivation, supergravity was born, the underlying supersymmetry invariance being associated with a spin-3/2 fermionic partner (the 'gravitino') for the bosonic spin-2 graviton. Moreover, since supersymmetry requires very special types of matter, such a scheme lends credence to the claim that a successful theory of quantum gravity must 
involve unifying the fundamental forces; i.e., the extra fields needed to cancel the graviton infinities might be precisely those associated with some grand unified scheme.

Recall from Section 3.1 that the mark of unification of two forces is the equality of their coupling constants; and that the energy-dependence of the coupling constants for the electromagnetic, weak and strong nuclear forces renders them at least approximately equal at around $10^{20} \mathrm{Mev}$. Here, it is of considerable interest to note that the introduction of supersymmetry greatly improves the prospects for exact equality: there is every expectation that these three fundamental forces do unify exactly in the supersymmetric version of the theory. The fact that $10^{20} \mathrm{Mev}$ is not that far away from the Planck energy $\left(10^{22} \mathrm{Mev}\right)$ adds further weight to the idea that a supersymmetric version of gravity may be needed to guarantee its inclusion in the pantheon of unified forces.

Early expectations for supergravity theory were high following successful low-order results, but it is now generally accepted that if higher-loop calculations could be performed (they are very complex) intractable divergences would appear once more. However, this line of thought continues and the torch is currently carried by the superstring programme, which in terms of numbers of papers produced per week is now by far the dominant research programme in quantum gravity.

The superstrings programme has had two phases. The first phase began in earnest in the mid-1980s (following seminal work in the mid 1970s) and used a perturbative approach; as we shall see, its treatment of spacetime can be presented readily enough in terms of our four probes listed in Section 4.1. The second phase began in the early 1990s, and 'still rages'. It has yielded rich insights into the underlying non-perturbative theory. But the dust has by no means settled. Even the overall structure of the underlying theory remains very unclear; and in particular, its treatment of spacetime is too uncertain for our four probes to be applied. Accordingly, our discussion of the second phase will forsake the probes, and just report how recent developments indicate some fundamental limitations in the manifold conception of spacetime. ${ }^{43}$

\footnotetext{
${ }^{43}$ For more detailed discussion of superstrings, see Part 4 of this volume.
} 


\subsubsection{Perturbative superstrings}

The perturbative superstrings programme involves quantising a classical system; but the system concerned is not general relativity, but rather a system in which a one-dimensional closed string propagates in a spacetime $\mathcal{M}$ (whose dimension is in general not 4). More precisely, the propagation of the string is viewed as a map $X: \mathcal{W} \rightarrow \mathcal{M}$ from a two-dimensional 'world-sheet' $\mathcal{W}$ to spacetime $\mathcal{M}$ (the 'target spacetime'). The quantisation procedure quantises $X$, but not the metric $\gamma$ on $\mathcal{M}$, which remains classical. The appropriate classical theory for the simplest such system is described by the famous Polyakov action; which is invariant under conformal transformations on $\mathcal{W}$. To preserve this conformal invariance in the quantised theory, and to satisfy other desirable conditions, the following conditions are necessary:

1. the theory is made supersymmetric;

2. the spacetime $\mathcal{M}$ has a certain critical dimension (the exact value depends on what other fields are added to the simple bosonic string); and

3. the classical spacetime metric $\gamma$ on $\mathcal{M}$ satisfies a set of field equations that are equivalent to the (supergravity version of) Einstein's field equations for general relativity plus small corrections of Planck size: this is the sense in which general relativity emerges from string theory as a low-energy limit. ${ }^{44}$

Superstring theory has the great advantage over the particle-physics programme that, for certain string theories, the individual terms in the appropriate perturbation expansion are finite. Furthermore, the particle content of theories of this type could be such as to relate the fundamental forces in a unified way. Thus these theories provide a concrete realisation of the old hope that quantum gravity necessarily involves a unification with the other fundamental forces in nature.

\footnotetext{
${ }^{44}$ The more realistic superstring theories involve an additional massless 'dilaton' scalar field $\phi$, and a massless vector particle described by a three-component field strength $H_{\mu \nu \rho}$. The presence of these extra fundamental fields has a major effect on the classical solutions of the field equations; in particular, there have been many studies recently of black-hole and cosmological solutions.
} 


\subsubsection{Spacetime according to the Perturbative Superstrings Pro- gramme}

The response given by the perturbative superstrings programme to the four conceptual probes in Section 4.1 is broadly as follows.

1. Use of standard quantum theory. As in the case of the particle-physics programme, the basic technical ideas of standard quantum theory are employed, albeit with suitable adaptations to handle the gauge structure of the theory. Similarly, the Copenhagen interpretation of the theory is, arguably, applicable; namely, by using as the required classical framework or background structure, the solution of the low-energy field equations for the spacetime metric $\gamma$. On the other hand, one can also argue contrariwise, that it is unsatisfactory to have the interpretation of our fundamental quantum theory only apply in a special regime, viz. low energies.

And as for the particle-physics programme, other interpretations of quantum theory discussed in Section 2.1 might be applicable. But they have not been developed in connection with superstrings, and so far as we can see, perturbative superstring theory gives no special reason in favour of them.

2. Use of standard spacetime concepts. In perturbative superstring theories, the target spacetime $\mathcal{M}$ is modelled using standard differential geometry, and there seems to be no room for any deviation from the classical view of spacetime. However, in so far as the dimension of $\mathcal{M}$ is greater than four, some type of 'Kaluza-Klein' scenario is required in which the extra dimensions are sufficiently curled up to produce no perceivable effect in normal physics, whose arena is a 4-dimensional spacetime.

3. The spacetime diffeomorphism group. Superstring theory shows clearly how general relativity can occur as a fragment of a much larger structurethereby removing much of the fundamental significance formerly ascribed to the notions of space and time. True, the low-energy limit of these theories is a form of supergravity but, nevertheless, standard spacetime ideas do not play a central role. This is reflected by the graviton being only one of an infinite number of particles in the theory. In particular, the spacetime diffeomorphism group $\mathcal{D}$ appears only as part of a much bigger structure, as in option (ii) of the discussion in Section 3.2. Consequently, its technical importance for the quantum scheme is largely subsumed by the bigger group.

4. The problem of time The perturbative expansion in a superstring theory 
takes place around the background given by the solution to the low-energy field equations for the spacetime metric $\gamma$. In particular, this provides a background causal structure; and hence - in that sense - there is no problem of time.

On the other hand, the situation is similar in many respects to that of the particle-physics programme; in particular, there are the same worries about the meaning of 'causality' and 'time' in any precise sense. There is, however, an important difference between superstring theory and the simple perturbative quantisation of general relativity-namely, there are realistic hopes that a proper non-perturbative version of the former is technically viable. If such a theory is found it will be possible to address the conceptual problems with time and causality in a direct way-something that is very difficult in the context of the (mathematically non-existent!) theory of perturbatively quantised general relativity.

\subsubsection{The Second Phase of Superstrings}

Since the early 1990s, a lot of work in the superstrings programme has focussed on exploring the underlying non-perturbative theory. These developments seem to have striking implications for our conception of space and time at the Planck scale. So now we turn to these, albeit with trepidation, since the dust has by no means yet settled in this area of research; and what the implications are is far from clear!

These developments are based on various types of 'duality' transformation or symmetry. For example, one of the simplest forms of duality (' $T$ duality') arises when the target space is a five-dimensional manifold of the form $\mathcal{M}_{4} \times S^{1} ;\left(S^{1}\right.$ is the circle). It transpires that the physical predictions of the theory are invariant under replacement of the radius $R$ of the fifth dimension with $2 \alpha^{\prime} / R$. Thus we cannot differentiate physically between a very small, and a very large, radius for the additional dimension-indeed, there is a precise sense in which they are 'gauge' equivalent to each other. One of the most important implications of this invariance is that there exists a minimum length of $R_{\min }=\sqrt{2} \alpha^{\prime},{ }^{45}$ - an idea that must surely have significant implications for our overall understanding of the conceptual implications of the theory.

\footnotetext{
${ }^{45}$ The phenomenon can be generalised to more than one extra dimension and with a topology that is more complex than just a product of circles.
} 
Another type of duality (' $S$-duality') involves the idea that, for certain theories the physics in the large-coupling limit is given by the weak-coupling limit of a 'dual' theory whose fundamental entities can be identified with solitonic excitations of the original theory. It is believed that the several known consistent perturbative superstring theories are related in this way to each other $^{46}$ and also to the theories involving extended objects ('membranes') of dimension greater than one. Ideas of this type are certainly attractive, not least because they provide a real possibility of theoretically probing the physically interesting, high-energy regimes of such theories.

In short, these developments suggest rather strongly that the manifold conception of spacetime is not applicable at the Planck length; but is only an emergent notion, approximately valid at much larger length scales. We shall take up this idea, in general terms, in Section 5. At a more technical level, the new ideas suggest that Lagrangian field-theoretic methods (which are used in the perturbative superstring theories) are reaching the limit of their domain of applicability, and should be replaced by - for example - a more algebraic approach to theory construction that places less reliance on an underlying classical system of fields; (i.e., the third of the four types of approach we listed in Section 3.1.3).

\subsection{The Canonical Quantum Gravity Programme}

\subsubsection{Quantum Geometrodynamics}

The canonical approach to quantum gravity starts with a reference foliation of spacetime with respect to which the appropriate canonical variables are defined. ${ }^{47}$ These are the 3 -metric $g_{a b}(x)$ on a spatial slice $\Sigma$ of the foliation, and a canonical conjugate $p^{a b}(x)$ that - from a spacetime perspective -is related to the extrinsic curvature of $\Sigma$ as embedded in the four-dimensional spacetime.

A key property of general relativity - which reflects the role of the group $\mathcal{D}$ of spacetime diffeomorphisms - is that these variables are not independent, but instead satisfy certain constraints, usually written as

$$
\mathcal{H}_{a}(x)=0
$$

\footnotetext{
${ }^{46} \mathrm{~A}$ third type of duality known as 'mirror symmetry' also plays an important role here.

${ }^{47} \mathrm{~A}$ fairly comprehensive bibliography of papers on canonical general relativity can be found in the review papers [28, 29].
} 


$$
\mathcal{H}_{\perp}(x)=0
$$

where $\mathcal{H}_{a}(x)$ and $\mathcal{H}_{\perp}(x)$ are complicated functions of the $g$ and $p$, and their derivatives.

The constraint functions $\mathcal{H}_{a}$ and $\mathcal{H}_{\perp}$ play a fundamental role in the theory since their Poisson bracket algebra (known as the 'Dirac algebra') is that of the group $\mathcal{D}$ of spacetime diffeomorphisms projected along, and normal to, the spacelike hypersurfaces of the reference foliation. Thus the basic question of understanding the role of spacetime diffeomorphisms is coded in the structure of these constraints.

In addition to the constraint equations Eqs. (4-5), there is also a collection of dynamical equations that specify how the canonical fields $g_{a b}(x)$ and $p^{c d}(x)$ evolve with respect to the time variable associated with the given foliation. However, it transpires that these equations are redundant since it can be shown that if $\gamma$ is a spacetime metric on $\mathcal{M}$ that satisfies the constraint equations Eqs. (4-5) on any spacelike hypersurface, then necessarily the projected canonical variables $g_{a b}(x)$ and $p^{c d}(x)$ will satisfy the dynamical equations. In this sense, the entire theory is already coded into just the four constraint equations Eqs. (4-5); so, in practice, attention is almost invariably focussed on them alone. Furthermore, among these equations, Eq. (5) is the crucial one, essentially because - when viewed from a spacetime perspective $-\mathcal{H}_{\perp}$ is associated with the canonical generators of displacements in time-like directions.

This system can be quantised in a variety of ways. One possibility is to impose a gauge for the invariance associated with the Dirac algebra; solve the constraints Eqs. (4-5) classically; and then quantise the resulting 'true' canonical system in a standard way. However, the final equations are intractable in anything other than a perturbative sense, where they promptly succumb to virulent ultraviolet divergences.

Most approaches to canonical quantum gravity do not proceed in this way. Instead, the full set of fields $\left(g_{a b}(x), p^{c d}(x)\right)$ is quantised via the 'canonical commutation relations'

$$
\begin{aligned}
{\left[\widehat{g}_{a b}(x), \widehat{g}_{c d}\left(x^{\prime}\right)\right] } & =0 \\
{\left[\widehat{p}^{a b}(x), \widehat{p}^{c d}\left(x^{\prime}\right)\right] } & =0 \\
{\left[\widehat{g}_{a b}(x), \widehat{p}^{c d}\left(x^{\prime}\right)\right] } & =i \hbar \delta_{(a}^{c} \delta_{b)}^{d} \delta^{(3)}\left(x, x^{\prime}\right)
\end{aligned}
$$

of operators defined on the 3 -manifold $\Sigma$. Following Dirac, the constraints are 
interpreted as constraints on the allowed state vectors $\Psi$, so that $\widehat{\mathcal{H}}_{a}(x) \Psi=$ $0=\widehat{\mathcal{H}}_{\perp}(x)$ for all $x \in \Sigma$. In particular, on choosing the states as functions of the three-geometry $g$-and with operator representatives $\left(\widehat{g}_{a b}(x) \Psi\right)[g]:=$ $g_{a b}(x) \Psi[g]$ and $\left(\widehat{p}^{c d}(x) \Psi\right)[g]:=-i \hbar \delta \Psi[g] / \delta g_{a b}(x)$ - the constraints $\widehat{\mathcal{H}}_{a} \Psi=0$ imply that $\Psi[g]$ is constant under changes of $g$ induced by infinitesimal diffeomorphisms of the spatial 3-manifold $\Sigma$; and the crucial constraint $\widehat{\mathcal{H}}_{\perp}(x) \Psi=$ 0 becomes the famous Wheeler-DeWitt equation.

But the Wheeler-DeWitt equation is horribly ill-defined in any exact mathematical sense and, unfortunately, perturbative approaches to its definition and solution are as virulently badly behaved as are its particle-physics based cousins: in both cases the problem is trying to define products of operator fields defined at the same point. Indeed, until the rise of the Ashtekar programme (see below), most of the work developing and using the WheelerDeWitt equation in anything other than a totally formal sense relied on truncating the gravitational field to just a few degrees of freedom, so that it becomes a partial differential equation in a finite number of variables, which one can at least contemplate attempting to solve exactly. ${ }^{48}$

In this context, we should mention again the Euclidean programme in quantum gravity. Here, the central role is played by functional integrals over all the Riemannian - rather than Lorentzian - metrics on a four-dimensional manifold $\mathcal{M}$. (The motivation for Riemannian metrics is partly an analogy with the successful use of imaginary time in Yang-Mills theory.) It can be shown that the functional $\Psi[g]$ of $g$ defined by certain such functional integrals satisfies (at least, in a heuristic way) the Wheeler-DeWitt equation; in particular, this is the basis of the famous Hartle-Hawking 'no boundary' proposal for the 'wave-function of the universe' in quantum cosmology. So in this sense, the Euclidean programme amounts to a way of constructing states for quantum geometrodynamics. For this reason, and also because we discuss this programme (and its use in quantum cosmology) in detail elsewhere [1], we set it aside here. ${ }^{49}$

\footnotetext{
${ }^{48}$ So one should not attach too much weight to the results of such simple approximations. Admittedly, models of this type can be valuable tools for exploring the many conceptual problems that arise in quantum cosmology. [30].

${ }^{49} \mathrm{~A}$ convenient recent source for many of the original articles is Gibbons and Hawking
} 


\subsubsection{The Ashtekar programme}

As emphasised above, the Wheeler-DeWitt equation is ill-defined in any exact mathematical sense. However, a major advance took place in 1986 when Ashtekar [31] found a set of canonical variables which produce a dramatic simplification of the structure of the central constraint functions $\mathcal{H}_{a}(x)$ and $\mathcal{H}_{\perp}(x)$. Since then there has been a very active programme to exploit these new variables, both in classical general relativity and in quantum gravity.

From a technical perspective, one of the great dangers in canonical quantum gravity is the generation of anomalous quantum excitations of nonphysical modes of the gravitational field. However, even to talk of such things requires the operators to be defined rigorously - a task that is highly nontrivial, since this is the point at which the infamous ultraviolet divergences are likely to appear. One of the main reasons why the Ashtekar programme is potentially so important is the hope it offers of being able to define these operators properly, and hence address such crucial issues as the existence of anomalous excitations.

The developments in the last decade have been very impressive and, in particular, there is now real evidence in support of the old idea that nonperturbative methods must play a key role in constructing a theory of quantum gravity. If successful in its current form, this programme will yield a theory of quantum gravity in which unification of the forces is not a necessary ingredient. Thus the Ashtekar programme demonstrates the importance of distinguishing between a quantum theory of gravity itself, and a 'theory of everything' which of necessity includes gravity.

In spite of their great structural significance, the use of the Ashtekar variables has had little impact so far on the conceptual problems in canonical quantum gravity, and so we shall not discuss the technical foundations of this programme here. However, it is important to note that one of the new variables is a spin-connection, which suggested the use of a gravitational analogue of the gauge-invariant loop variables introduced by Wilson in YangMills theory. Seminal work in this area by Rovelli and Smolin [32] has produced many fascinating ideas, including a demonstration that the area and volume of space are quantised - something that is evidently of philosophical interest and which has no analogue in quantum geometrodynamics. We shall return to these ideas briefly in Section 5 . 


\subsubsection{Spacetime According to the Canonical Quantum Gravity Programme}

The response given by the canonical quantum gravity programme to the four probes in Section 4.1 is broadly as follows.

1. Use of standard quantum theory. The basic technical ideas of standard quantum theory are employed, suitably adapted to handle the non-linear constraints satisfied by the canonical variables. On the other hand, the traditional, Copenhagen interpretation of quantum theory is certainly not applicable unless a background spatial metric is assumed, and a resolution is found of the problem of time, at least at some semi-classical level. However, most attempts to implement the canonical scheme abhor the introduction of any type of background metric, and hence major conceptual problems can be expected to arise if this programme is ever fully realised.

Of course, one radical strategy for coping with this situation is 'to make a virtue of necessity', as discussed in Section 2.1 .3 ; i.e., to adopt the pilot-wave interpretation of quantum theory, and thereby introduce a background metric in a strong sense, involving a preferred foliation of spacetime. Note, however, that with its emphasis on configuration space, this interpretation would not seem appropriate for the Ashtekar programme where, unlike quantum geometrodynamics, the states are not functions on the configuration space of all 3-metrics.

Although we are setting aside quantum cosmology, we should add that since most work in quantum cosmology has been done within the canonical quantum gravity programme, there is another sense in which the Copenhagen interpretation is certainly not applicable to canonical quantum gravity; whereas (as discussed in Section 2.1.2), rivals such as Everettian interpretations might be.

2. Use of standard spacetime concepts. In this regard, canonical quantum gravity in effect 'lies between' the conservatism of the particle-physics programme, and the radicalism of superstrings. For like the former, it uses a background dimensional manifold (but it uses no background metric). More precisely, the canonical theory of classical relativity assumes ab initio that the spacetime manifold $\mathcal{M}$ is diffeomorphic to $\Sigma \times \mathbb{R}$ where $\Sigma$ is some 3manifold; and this 3-manifold becomes part of the fixed background in the quantum theory - so that, as for the particle-physics programme, there is no immediate possibility of discussing quantum changes in the spatial topology. 
3. The spacetime diffeomorphism group. In the canonical quantum gravity programme, the classical Poisson-bracket algebra of the constraint functions (i.e., the Dirac algebra) can be interpreted as the algebra of spacetime diffeomorphisms projected along, and normal to, spacelike hypersurfaces. In the quantum theory, it is usually assumed that this Poisson-bracket algebra is to be replaced with the analogous commutator algebra of the corresponding quantum operators

The Dirac algebra contains the group of spatial diffeomorphisms, Diff $(\Sigma)$, as a subgroup but it is not itself a genuine group. Invariance under $\operatorname{Diff}(\Sigma)$ means that the functionals of the canonical variables that correspond to physical variables are naturally construed as being non-local with respect to $\Sigma$. Recent work with the loop-variable approach to canonical quantum gravity has been particularly productive in regard to the implications of invariance under spatial diffeomorphisms.

The role of the full Dirac algebra is more subtle and varies according to the precise canonical scheme that is followed. There are still contentious issues in this area-particularly in regard to exactly what counts as an 'observable' in the canonical scheme.

4. The problem of time. One of the main aspirations of the canonical approach to quantum gravity has always been to build a formalism with no background spatial, or spacetime, metric; (this is particularly important, of course, in the context of quantum cosmology). In the absence of any such background structure, the problem of time becomes a major issue.

There are various obvious manifestations of this. One is that the WheelerDeWitt equation makes no apparent reference to time, and yet this is to be regarded as the crucial 'dynamical' equation of the theory! Another manifestation concerns the starting canonical commutation relations Eqs. (6-8). The vanishing of a commutator like Eq. (6) would normally reflect the fact that the points $x$ and $x^{\prime}$ are 'spatially separated'. But what does this mean in a theory with no background causal structure?

The situation is usually understood to imply that, as mentioned in Section 3.3.2 (strategies 2 and 3), 'time' has to be reintroduced as the values of special physical entities in the theory - either gravitational or material - with which the values of other physical quantities are to be correlated. Thus, rather than talking about clocks measuring time - which suggests there is some external temporal reference system - we think of time as being defined by a clock, 
which in this case means part of the overall system that is being quantised. Thus physical time is introduced as a reading on a 'physical' clock.

Unfortunately, it is a major unsolved problem whether (i) this can be done at all in an exact way; and (ii) if so, how the results of two different such choices compare with each other, and how this is related to spatiotemporal concepts. In fact, there are good reasons for thinking that it is not possible to find any 'exact' internal time, and that the standard notion of time only applies in some semi-classical limit of the theory. In this way time would be an emergent or phenomenological concept, rather like temperature or pressure in statistical physics. We discuss this line of thought further in Section 5.4 of [1]; here we just emphasise that it is specifically about time, not spacetime - and in that sense, not this paper's concern. Section 5 will discuss the idea that spacetime, though not distinctively time, is phenomenological.

\section{Towards Quantum Spacetime?}

\subsection{Introduction: Quantisation and Emergence}

In this Section, we turn to discuss some treatments of spacetime that are in various ways more radical than those given by the programmes in Section 4 . We shall adopt the classification in Section 3.1.3 of four types of approach to quantum gravity. Recall that they were:

(1) quantising general relativity;

(2) quantising a different classical theory, while still having general relativity emerge as a low-energy (large-distance) limit;

(3) having general relativity emerge as a low-energy limit of a quantum theory that is not a quantisation of a classical theory; and finally, and most radically,

(4) having both general relativity and quantum theory emerge from a theory very different from both.

Thus in Section 5.2, we will discuss type (2); in Section 5.3, type (3); and in Section 5.4, type (4). But it will help set these discussions in context, to take up two topics as preliminaries: (i) the relation of the programmes in Section 
4 to this classification; and (ii) the notion of emergence, and its relation to quantisation, in general.

\subsubsection{Some Suggestions from the Three Programmes}

It is easy to place the three programmes in Section 4 within the above classification. We have seen that the particle-physics and canonical programmes are examples of type (1), while the superstrings programme is an example of type (2) (at least in its perturbative version). But we should add two remarks to 'sketch in the landscape' of this classification.

1. All three programmes are similar in that the main way they go beyond what we called the common treatment of spacetime of our "ingredient theories' (viz. as a 4-dimensional manifold with a (classical) Lorentzian metric), is by quantizing a quantity that is a standard type of physical variable within the context of classical physics defined using the familiar tools of differential geometry. For the particle-physics and canonical programmes (Sections 4.3, 4.5), this is the spatiotemporal metric $\gamma$, and the 3-metric $g$ respectively. In the perturbative superstrings programme, the variable concerned is the function $X$ that maps a loop into the target spacetime. However, in this Section, we will discuss treatments that in some way or other 'go beyond' quantizing standard classical objects.

2. On the other hand, we should stress that the two main current programmes discussed in Section 4 make various radical suggestions about spacetime: suggestions which are not reflected by their being classified as types (1) and (2) in the schema above. We already saw some of these suggestions in Section 4. In particular, we saw that the superstrings programme requires spacetime to have a dimension which is in general not four; and - more strikingly - recent work on non-perturbative approaches suggests that more than one manifold may contribute at the Planck scale, or that models based on 'non-commutative geometry' may be appropriate. And for the canonical quantum gravity programme, we mentioned the discrete spectra of the spatial area and spatial volume quantities: results that arguably suggest some type of underlying discrete structure of space itself. 
But these programmes also make other such suggestions. For example, we will see in Section 5.2 a general way in which quantising a metric (as in these programmes) suggests a quantisation of logically weaker structure such as differential or topological structure; these are called 'trickle-down effects'.

To sum up: Both these programmes threaten the ingredient theories' common treatment of spacetime, quite apart from their quantising the metric; indeed they even threaten the manifold conception of space or spacetime.

\subsubsection{Emergence and Quantisation}

At this point it is worth developing the contrast (introduced in Section 1.1) between these two general strategies which one can adopt when attempting to go beyond the common treatment of spacetime. The distinction can be made in terms of any part of the common treatment, not just metrical structure on which the programmes in Section 4 focus: for example, topological structure.

To 'go beyond' such a structure, one strategy is to argue that it is emergent (in physics' jargon: 'phenomenological'). 'Emergence' is vague, and indeed contentious; for along with related notions like reduction, it is involved in disputes, central in philosophy of science, about relations between theories and even sciences. But here, we only need the general idea of one theory $T_{1}$ being emergent from another $T_{2}$ if in a certain part of $T_{2}$ 's domain of application (in physics' jargon, a 'regime': usually specified by certain ranges of values of certain of $T_{2}$ 's quantities), the results of $T_{2}$ are well approximated by those of $T_{1}$-where 'results' can include theoretical propositions as well as observational ones, and even 'larger structures' such as derivations and explanations.

This relation of emergence can of course be iterated, yielding the idea of a 'tower' of theories, each emerging from the one above it. Figure 1 portrays this idea, for the case of interest to us, viz. where the 'bottom theory' is classical general relativity. Figure 1 also uses the physics jargon of a theory being 'phenomenological'; and for the sake of definiteness, it assumes an uppermost 'ultimate' theory - an assumption to which, as is clear from Section 1.3, we are not committed. We should add that of course many different towers will in general branch off from a given theory. ${ }^{50}$

\footnotetext{
${ }^{50}$ For more discussion of emergence, especially in relation to reduction, see Section 2 of our complementary essay [1].
} 
The 'ultimate' theory

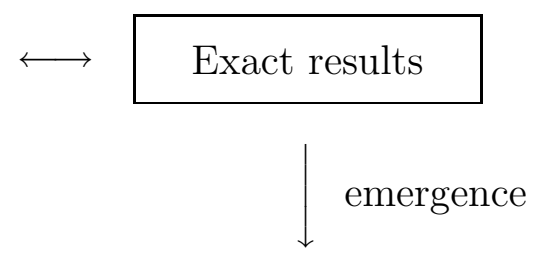

Phenomenological theory

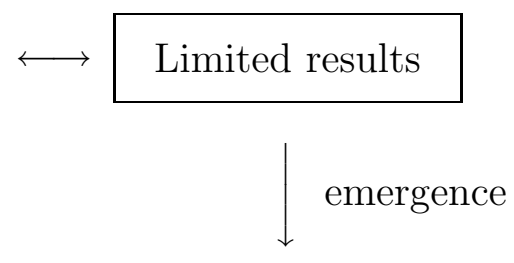

Phenomenological theory

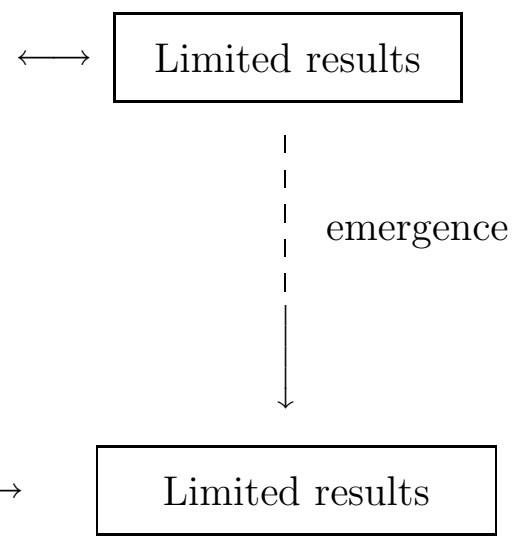

Figure 1. A hierarchy of phenomenological theories

So much for the general idea of emergence. The other strategy for 'going beyond a classical structure' is to try to quantize it, in some sense; and then to recover it as some sort of classical limit of the ensuing quantum theory. We say 'quantize in some sense', because although 'quantization' is certainly less vague than 'emergence', it is far from being precise. There is an open-ended family of 'quantization procedures' that are only provably equivalent on some simple cases (such as certain finite-dimensional, unconstrained Hamiltonian systems); and there is no procedure known whereby an arbitrary classical system can be quantised in an unequivocal way. Similarly, we say 'some sort of classical limit', because (as we said in Section 3.1.3) 'classical limit' also has various senses: it can refer to special states whose evolution over time follows classical laws, or to certain quantum quantities taking values in a 
range where classical theory is successful.

Though these two strategies are vague, the general idea of them is enough to make it clear that they are independent. That is: neither implies the other, though of course they can be combined; (as the classification of approaches (1)-(4) makes clear).

Thus one can maintain that some classical structure is emergent, without quantizing it (i.e., exhibiting it as a classical limit of a quantisation). Indeed, there are at least two ways one can do this. The first is familiar from our discussion of superstrings (Section 4.4): a classical structure (there, the metric geometry of general relativity) can emerge from a quantum theory (superstrings) which is not a quantized version of a classical theory of the structure. The second way is independent of quantum theory: viz. a classical structure could emerge from some theory which has nothing to do with quantum theory. (In the context of quantum gravity, this way is taken by approach (4) in the classification of Section 3.1.3.)

The converse implication can also be questioned. Quantizing a structure does not ipso facto render the original classical structure emergent. Agreed, if we quantize a structure, then we can investigate the resulting quantum theory's classical limits: and even allowing for the vagueness of 'quantisation' and 'classical limit', we are more or less guaranteed to be able to identify the original classical structure as such a limit, or as a feature of such a limit. But in view of the vagueness of 'emergence', this might not count as showing the classical structure to be emergent within the quantum theory. Indeed, quite apart from subtleties about the philosophical notion of 'emergence', the measurement problem of quantum theory looms over the interpretation of such limits as 'recovering the classical world'.

So much by way of general discussion of these two strategies, and their independence. The upshot, for our topic of how to depart more radically from the common treatment of spacetime than 'just' by quantizing metrical structure, is that one can apply either or both of these two strategies to other classical structures - for example to topological structure. We will see such applications in more detail in the next three Subsections' discussions of approaches (2)-(4) respectively.

Before embarking on those discussions, we should enter two caveats. First: the ideas we are about to discuss are far less established and understood, than are the trio of bona fide research programmes in Section 4; so our discussion needs must be much more tentative. Second: we will downplay the first 
strategy, i.e., emergence, on the grounds that our complementary paper [1] discusses it - both in general philosophical terms, and (for classical spacetime structure), in terms of classical limits of a theory of quantum gravity. Also very little is known about the prospects for this strategy used on its own, i.e., uncombined with the second strategy of quantisation - as we will see in Sections 5.3 and 5.4 .

\subsection{Quantisation 'Below' the Metric}

We turn to discuss approach (2), where one quantises a theory other than classical general relativity, but obtains it as a low-energy limit of the quantised theory. Needless to say, only a tiny fraction of the vast range of classical theories has been quantised, so that in full generality, little is known about this approach; and much of what is known concerns the superstrings programme.

So in this Subsection, we will confine ourselves to general comments about the treatment of spacetime to be expected once one adopts this approach. Furthermore, we will only consider applying this approach to quantising classical spacetime structures; (hence this Subsection's title). Given this restriction, our discussion is given a natural structure by the fact that the common treatment of spacetime - viz. as a pair $(\mathcal{M}, \gamma)$, with $\mathcal{M}$ a 4-dimensional differentiable manifold, and $\gamma$ a Lorentzian metric - appears at one end of a hierarchical chain of structure; so that we can picture the introduction of a quantum effect 'below the metric $\gamma$ ' in terms of an earlier point in the chain. Of course, a given mathematical structure can often be placed in more than one such chain, and the question then arises of which chain to use.

The common treatment of spacetime as a pair $(\mathcal{M}, \gamma)$ fits naturally into the chain

$$
\text { set of spacetime points } \rightarrow \text { topology } \rightarrow \text { differential structure } \rightarrow(\mathcal{M}, \gamma)
$$

Indeed, this chain is implicit in much of our previous discussion. The bottom level is a set $\mathcal{M}$, whose elements are to be identified with spacetime points; but this set is formless, its only general mathematical property being its cardinal number. In particular, there are no relations between the elements of $\mathcal{M}$, and no special way of labelling any such element. The next step is to impose a topology on $\mathcal{M}$ so that each point acquires a family of neighbourhoods. Then one can talk about relationships between points, albeit in a 
rather non-physical way. This defect is overcome by adding the key ingredient of the conventional treatment of spacetime: the topology of $\mathcal{M}$ must be compatible with that of a differentiable manifold, so that a point in $\mathcal{M}$ can be labelled uniquely (at least, locally) by giving the values of four real numbers. In the final step a Lorentzian metric $\gamma$ is placed on $\mathcal{M}$, thereby introducing the ideas of the lengths of a path joining two spacetime points, parallel transport with respect to a Riemannian connection, causal relations between pairs of points etc. Note that an analogous discussion applies to the usual modelling of space and time individually by a 3-dimensional, and 1-dimensional manifold respectively.

Note that a variety of intermediate stages can be inserted: for example, the link 'differential structure $\rightarrow(\mathcal{M}, \gamma)$ ' could be factored as

$$
\text { differential structure } \rightarrow \text { causal structure } \rightarrow(\mathcal{M}, \gamma)
$$

A quite different scheme arises by exploiting the fact that a differentiable manifold $\mathcal{M}$ is uniquely determined by the algebraic structure of its commutative ring of differentiable functions, $\mathcal{F}(\mathcal{M})$ (cf. our discussion in Section 2.2 of the spectral theorem for commutative $\mathrm{C}^{*}$ algebras.) And a ring is itself a complicated algebraic structure that can be analysed into a hierarchy of substructures in several ways. Thus one alternative chain to Eq. (9) is

$$
\text { set } \rightarrow \text { abelian group } \rightarrow \text { vector space } \rightarrow \mathcal{F}(\mathcal{M}) \rightarrow(\mathcal{M}, \gamma) \text {. }
$$

Given these chains of structure leading to $(\mathcal{M}, \gamma)$, and others like them, it is clear what are the options facing approach (2). One has to decide:

(i) which of the chains to $(\mathcal{M}, \gamma)$ to use; and

(ii) at what level in the chosen chain to try to quantise.

For example, if one uses the first chain, one faces such questions as: should we accept a fixed set of spacetime (or, for a canonical approach: spatial) points, but let the topology and/or differential structure be subject to quantum effects? Or should we say that the notion of a spacetime point itself is not meaningful at a fundamental level: i.e., it is a concept that should not appear in the theory, even in a mathematical sense?

We end by making two general comments about this situation, corresponding to the two decisions, (i) and (ii). 
In regard to (i), we stress that the details of one's programme will depend strongly on the initial decision about which chain to use. Thus if one decides to apply quantization to the second chain Eq. (11), one is led naturally to consideration of the algebraic approach to classical general relativity pioneered by Geroch [33] ('Einstein algebras') and non-commutative analogues thereof [34]. And, of course, the idea of a non-commutative version of the algebra $\mathcal{F}(\mathcal{M})$ was one of the motivating factors behind Connes' seminal ideas on non-commutative geometry [35].

Concerning (ii), one should be aware that, once a chain has been chosen, quantisation at one level could 'trickle down' to produce quantum effects at a 'lower', more general, level in the chain; (i.e. to the left in our diagrams). For example, quantisation of the metric could trickle down to the differential structure or topology. ${ }^{51}$

Such 'trickle-down' effects were envisaged by Wheeler [36] in his original ideas about quantum topology in the context of canonical quantization. His idea was that large quantum fluctuations in a quantized 3-metric $\hat{g}_{a b}(x)$ would generate changes in the spatial topology; for the effects of quantum gravity would become more pronounced at decreasing distances, resulting eventually in a 'foam like' structure at around the Planck length.

We stress that such effects depend on an appropriate mechanism. Thus, contra Wheeler's intuition that quantum gravity effects become stronger at decreasing distances, one might hold that, in fact, quantum gravity is 'asymptotically-free' - so that the effects become smaller as the scale reduces. Under these circumstances, there would be no metric-driven topology changes. ${ }^{52}$

Another, more radical, example of trickle-down effects arises in connection with Penrose's thesis that a projective view of spacetime structure is more appropriate in quantum gravity: in particular, a spacetime point should be identified with the collection of all null rays that pass through it. Quantising the spacetime metric will then induce quantum fluctuations in the null rays, which will therefore no longer intersect in a single point. In this way, quantum fluctuations at the top of the first chain Eq. (9) trickle right down to the

\footnotetext{
${ }^{51}$ This was mentioned in Section 5.1.1, as a way in which the programmes in Section 4 put pressure on the manifold conception of spacetime; a way additional to those already discussed.

${ }^{52}$ The idea that gravity might be asymptotically-free was studied some years ago by Fradkin and Tseytlin [37] in the context of $R+R^{2}$ theories of gravity.
} 
bottom of the chain, so that the very notion of a 'spacetime point' acquires quantum overtones.

\subsection{Spacetime from a Non-Quantisation}

We turn to approach (3) in our classification, according to which general relativity emerges as a low-energy limit of a quantum theory that is not given as the quantisation of a classical theory, but rather 'intrinsically' in some way.

By and large, programmes following this approach will reject the conception of spacetime as a manifold ('from the outset', rather than 'sneaking up' on this conclusion in the way the programmes in Section 4 do). Agreed, the envisaged quantum theory might in principle include the postulation of a spacetime manifold (though it is not a quantisation of a classical theory defined on that manifold): a manifold which then turns out to be the manifold on which the low-energy limit, general relativity, is defined. But by and large, this circumstance would be odd: why should the quantum theory postulate just what the emergent approximation needs? Accordingly, we will here consider programmes (or, more precisely, mainly one fragment of one possible programme!) which do indeed reject the spacetime manifold at the fundamental level.

An example of the way the envisaged quantum theory might have general relativity as a low-energy limit is as follows. Return to the tower of theories of Section 5.2, and imagine that one result of emergence at some level in the tower is: (i) the idea of a 'local region' - not regarded as a subset of something called 'spacetime', but rather as an emergent concept in its own right; together with (ii) an algebra of such regions that specifies their theoretical use, and that can be identified mathematically as the algebra of a certain open covering of a genuine continuum manifold $\mathcal{M}$. Hence - as long as one keeps to the phenomena appropriate to this level-it is as if physics is based on the spacetime manifold $\mathcal{M}$, even though this plays no fundamental role in the 'ultimate' theory with which we started. Specific ideas of this type have arisen in the context of attempts to quantise the point-set topology of a set $[38,39]$. We note in passing that the mathematics of locales, and more generally topos theory (in particular, the idea of a 'Grothendieck topos') provides a natural framework in which to develop the idea that regions are more important than points. 
The possible significance of regions, rather than points, arises also in recent ideas about the nature of quantum physics in a bounded region. These go back to an old remark of Bekenstein [40] to the effect that any attempt to place a quantity of energy $E$ in a spatial region with boundary area $A$-and such that $E>\sqrt{A}$-will cause a black hole to form, and this puts a natural upper bound on the value of the energy in the region. The implication is that in any theory of quantum gravity whose semi-classical states contain something like black-hole backgrounds, the quantum physics of a bounded region will involve only a finite-dimensional Hilbert space. This intriguing possibility is closely related to the so-called 'holographic' hypothesis of t'Hooft [41] and Susskind [42] to the effect that physical states in a bounded region are described by a quantum field theory on the surface of the region, with a Hilbert space of states that has a finite dimension.

Ideas of this type could have major implications for quantum gravity. In particular - and in terms of the tower in Figure 1-the implication is that at one level of phenomenological theory the idea of local spacetime regions makes sense, and in those regions the quantum theory of gravity is finitedimensional. However, in the - possibly different-tower of phenomenological approximations that includes weak-field perturbative approaches to quantum gravity, the effective theory uses an infinite-dimensional Hilbert space to describe the states of weakly-excited gravitons. And, at this level, spacetime is modelled by a continuum manifold, with a full complement of spacetime points.

We should emphasise that an approach like this does not necessarily exclude the proposals of the more familiar research programmes in quantum gravity: such proposals would however become phenomenological, i.e., part of the emergence of general relativity and quantum theory. If our present understanding of quantum gravity is any guide, this effective quantisation of the gravitational field will involve a non-local-possibly string-like - structure. This raises the intriguing question of whether superstring theory and the loop-variable approach to canonical quantum gravity can both be regarded as different modes - or phases - of a more basic, common structure [43]. A central issue, presumably, is whether supersymmetry can be assigned some significant role in the Ashtekar programme. 


\subsection{Spacetime emergent from a Non-Quantum Theory}

Finally let us raise the question of the justification in quantum gravity of the use of standard quantum theory itself. So, in terms of Figure 1's portrayal of the emergence of general relativity, the idea now is that there would also be a tower of theories leading down to the emergence of standard quantum theory. Of course, in accordance with the point that towers can branch off from each other, this tower may well not be the same one as that leading to general relativity.

One particularly relevant issue in regard to quantum theory - and the only one that we shall discuss in any detail - is the question of what justifies its use of continuum concepts: specifically, its use of real and complex numbers. This question is very pertinent if one is already worried about the use of continuum ideas in the manifold model of space or time.

The formalism of quantum theory immediately suggests two answers: one concerning eigenvalues, and the other probabilities. Thus one might answer by saying that real numbers represent the possible results of measurements; (so that if eigenvalues of operators are to represent results, we want the operators to be self-adjoint). But why should measurement results be represented by real numbers? One natural, if not compelling, answer is that apparently all measurement results can in principle be reduced to the positions of a pointer in space - and space is modelled using real numbers. At the very least, this is certainly true of the elementary wave mechanics of a point particle moving in standard space; and this example, particularly the Hilbert space generalisation of its specific mathematical structure - has become one of the paradigms for quantum theory in general.

So according to this line of thought, the use of real numbers (and similarly: complex numbers) in quantum theory in effect involves a prior assumption that space should be modelled as a continuum. If so, then the suggestion that standard spacetime concepts break down at the scale of the Planck length and time, and must be replaced by some discrete structure which only 'looks like' a differentiable manifold at larger scales, means that we cannot expect to construct a theory of this discrete structure using standard quantum theorywith its real and complex numbers. Of course, this argument is not watertight; but it does illustrate how potentially unwarranted assumptions can enter speculative theoretical physics, and thereby undermine the enterprise.

The second possible answer to the question "what justifies quantum the- 
ory's use of real and complex numbers?' is that probabilities are real numbers between 0 and 1; (so that if probabilities are to be given by the squared norm of state vectors, the vector space must have $\mathbb{R}$ or $\mathbb{C}$ as its ground-field). But why should probabilities be represented by real numbers? Of course, if probability is construed as 'relative frequencies' of sequences of measurements, then the real numbers do arise naturally as the ideal limits of collections of rational numbers. However, the idea of measurement 'at the Planck length' is distinctly problematic (cf. our remarks in Section 1.3.3 about 'in principle inaccessibility'), and if the concept of probability is relevant at all in such regimes, one may feel that a different interpretation of this concept is more appropriate: for example, the propensity interpretation.

But there is no a priori reason why a 'propensity' (whatever that may mean!) should be modelled by a real number lying between 0 and 1. Agreed, it may well be appropriate sometimes to say that one propensity is 'larger' than another; but there may also be propensities that cannot be compared at all (a not unreasonable suggestion in the context of non-commuting operators in a quantum theory), and this suggests that a minimal model for such probabilities would be a partially-ordered set with some type of additional algebraic structure (so that 'sums' of probabilities can be defined for disjoint propositions).

For these reasons, a good case can be made that a complete theory of quantum gravity may require a revision of quantum theory itself in a way that removes the a priori use of continuum numbers in its mathematical formalism.

Finally, we note that, from time to time, a few hardy souls have suggested that a full theory of quantum gravity may require changing the foundations of mathematics itself. A typical argument is that standard mathematics is based on set theory, and certain aspects of the latter (for example, the notion of the continuum) are grounded ultimately in our spatial perceptions. However, our perceptions probe only the world of classical physics - and hence we feed into the mathematical structures currently used in all domains of physics, ideas that are essentially classical in nature. The ensuing category error can be remedied only by thinking quantum theoretically from the very outset - in other words, we must look for 'quantum analogues' of the categories of standard mathematics. 
How this might be done is by no means obvious. ${ }^{53}$ One approach is to claim that, since classical logic and set theory are so closely linked (a proposition $P$ determines - and is determined by - the class of all entities for which $P$ can be rightly asserted), one should start instead with the formal structure of quantum logic and try to derive an analogous 'non-Boolean set theory'. Such ideas are related to the exciting subject of topos theory, which can be viewed as a far-reaching generalisation of standard set theory. This is why, as mentioned in Section 5.3, topos theory is a natural arena within which to develop speculative schemes in which 'regions' of spacetime (or space, or time) are more important than 'points' (which may not exist at all). ${ }^{54}$

\subsection{Envoi}

Clearly, this Section has opened up a Pandora's box of possibilities for the overall shape of a theory of quantum gravity: possibilities that it is well-nigh impossible to adjudicate between - not least because it is very hard even to make individual possibilities precise and detailed. So we will make no pretence of judging them here.

Instead, by way of a modest and (mercifully brief!) conclusion, we want to stress some of this paper's lines of thought, that led to this Pandora's box. We began with the question how spacetime might be treated in a theory of quantum gravity. We described how the search for such a theory was beset by various conceptual difficulties, including difficulties about the ingredient theories, and about spacetime concepts - and also beset by a dire lack of data; (a predicament reviewed in Sections 1 to 3). On the other hand, we could not 'duck out' of searching for some such theory (Sections 3.1.1-3.1.2). We reviewed in Section 4 three main programmes that all proceed by quantising a classical theory which postulates a spacetime manifold. In various ways these programmes suggest that there are fundamental limitations to the applicability of the manifold conception of spacetime; (of course, they also have various more specific problems, both physical and conceptual). Thus our attention was turned to the more radical programmes of this Section ... where we admit to having to suspend judgment.

\footnotetext{
${ }^{53} \mathrm{~A}$ recent example of this type of thinking can be found in a book by Finkelstein[44].

${ }^{54}$ Topos theory has a related deep connection with non-standard logical structures: something we have exploited in recent work useing presheaf logic to analyse the KochenSpecker theorem in standard quantum theory $[45,46]$.
} 
To sum up: Quantum gravity is most unusual in comparison with other branches of physics, and indeed with most other branches of human enquiryor with other 'games people play'. It is an exciting unpredictable game, with

very few rules - and yet, as the sports commentators say, 'there is everything to play for!'

\section{Acknowledgements}

Chris Isham would like to thank the Mrs. L. D. Rope Third Charitable Settlement for financial support.

\section{References}

[1] J. Butterfield and C.J. Isham. On the emergence of time in quantum gravity. In J. Butterfield, editor, The Arguments of Time. Oxford University Press, Oxford, 1999. gr-qc/9901024.

[2] D. Kaiser. More Roots of Complementarity: Kantian Aspects and Influences. Studies in History and Philosophy of Science, 23:213-239, 1992.

[3] J. Butterfield. Worlds, minds and quanta. Aristotelian Society Supplementary Volume, 69:113-158, 1995.

[4] J. Butterfield. Whither the minds? British Journal for the Philosophy of Science, 47:200-221, 1996.

[5] K. Ridderbos. The loss of coherence in quantum cosmology. Studies in History and Philosophy of Modern Physics, 30B:41-60, 1999.

[6] J. Hartle. Spacetime quantum mechanics and the quantum mechanics of spacetime. In B. Julia and J. Zinn-Justin, editors, Proceedings of the 1992 Les Houches School, Gravitation and Quantisation, pages 285-480. Elsevier Science, 1995.

[7] C.J. Isham and N. Linden. Quantum temporal logic and decoherence functionals in the histories approach to generalised quantum theory. Journal of Mathematical Physics, 35:5452-5476, 1994. 
[8] A. Valentini. On the Pilot-Wave Theory of Classical, Quantum and Subquantum Physics. Springer-Verlag, Berlin, 2000.

[9] J. Bub. Interpreting the Quantum World. Cambridge University Press, Cambridge, 1997.

[10] G.C. Ghirardi, A. Rimini, and T. Weber. Unified dynamics for microscopic and macroscopic systems. Physical Review, D34:470-491, 1986.

[11] P. Pearle. Combining stochastical dynamical state-vector reduction with spontaneous localization. Physical Review, A39:2277-2289, 1989.

[12] P. Pearle and E. Squires. Gravity, energy conservation and parameter values in collapse models. 1995. quant-ph/9503019.

[13] J. Earman. Bangs, Crunches, Whimpers and Shrieks. Oxford University Press, Oxford, 1995.

[14] J. Earman and J. Norton. What price space-time substantivalism? The hole story. British Journal for the Philosophy of Science, 38:515-525, 1987.

[15] J. Earman. World Enough and Space-Time. MIT Press, Cambridge, Massachusetts, 1989.

[16] T. Jacobson. Thermodynamics of spacetime: The Einstein equation of state. Physical Review Letters, 75:1260, 1995.

[17] N. Bohr and L. Rosenfeld. Zur frage der messbarkeit der elektromagnetischen feldgrossen. Kgl. Danek Vidensk. Selsk. Math.-fys. Medd., 12:8, 1933.

[18] L. Rosenfeld. On quantization of fields. Nuclear Physics, 40:353-356, 1963.

[19] D.N. Page and C.D. Geilker. Indirect evidence for quantum gravity. Physical Review Letters, 47:979-982, 1981.

[20] R. Feynman. Lectures on gravitation. Acta Physical Polonica, XXIV:697, 1963. 
[21] D.G. Boulware and S. Deser. Classical general relativity derived from quantum gravity. Annals of Physics, 89:193, 1975.

[22] J. Donoghue. Perturbative dynamics of quantum general relativity. In Proceedings of the Eighth Marcel Grossmann Conference on General Relativity. 1998.

[23] K. Kuchař. Time and interpretations of quantum gravity. In Proceedings of the 4 th Canadian Conference on General Relativity and Relativistic Astrophysics, pages 211-314. World Scientific, Singapore, 1992.

[24] C.J. Isham. Canonical quantum gravity and the problem of time. In Integrable Systems, Quantum Groups, and Quantum Field Theories, pages 157-288. Kluwer Academic Publishers, London, 1993.

[25] W. Unruh and R.M. Wald. Time and the interpretation of quantum gravity. Physical Review, D40:2598-2614, 1989.

[26] C.J. Isham, R. Penrose, and D.W. Sciama. Quantum Gravity: An Oxford Symposium. Clarendon Press, 1975.

[27] C.J. Isham, R. Penrose, and D.W. Sciama. Quantum Gravity: A Second Oxford Symposium. Clarendon Press, 1981.

[28] C.J. Isham. Conceptual and geometrical problems in quantum gravity. In H. Mitter and H. Gausterer, editors, Recent Aspects of Quantum Fields, pages 123-230. Springer-Verlag, Berlin, 1992.

[29] K. Kuchař. Canonical quantum gravity. In R.J. Gleiser, C.N. Kozameh, and O.M. Moreschi, editors, General Relativity and Gravitation, 1992, pages 119-150. IOP Publishing, Bristol, 1993.

[30] M. Gell-Mann and J. Hartle. Classical equations for quantum systems. Physical Review, D47:3345, 1993.

[31] A. Ashtekar. New variables for classical and quantum gravity. Physical Review Letters, 57:2244-2247, 1986.

[32] C. Rovelli and L. Smolin. Loop space representation of quantum general relativity. Nuclear Physics, B331:80-152, 1990. 
[33] R. Geroch. Einstein algebras. Communications in Mathematical Physics, 25:271-275, 1972.

[34] G.N. Parfionov and R.R. Zapatrin. Pointless spaces in general relativity. International Journal of Theoretical Physics 34:717, 1995.

[35] A. Connes. Non Commutative Geometry. Academic Press, New York, 1994.

[36] J.A. Wheeler. Superspace and the nature of quantum geometrodynamics. In C. DeWitt and J.W. Wheeler, editors, Battelle Rencontres: 1967 Lectures in Mathematics and Physics, pages 242-307. Benjamin, New York, 1968.

[37] E.S. Fradkin and A.A. Tseytlin. Renormalizable asymptotically free quantum theory of gravity. Physics Letters, 104B:377-381, 1981.

[38] R.D. Sorkin. Finitary substitute for continuous topology. International Journal of Theoretical Physics, 30:923-947, 1991.

[39] C.J. Isham. An introduction to general topology and quantum topology. In H.C. Lee, editor, Physics, Geometry and Topology, pages 129-190. Plenum Press, New York, 1990.

[40] J.D. Bekenstein. The quantum mass spectrum of a Kerr black hole. Lettere a Nuovo Cimento, 11:467-470, 1974.

[41] G. t'Hooft. Dimensional reduction in quantum gravity. 1993 . grqc/9310026.

[42] L Susskind. The world as a hologram. Journal of Mathematical Physics, 36:6377-6396, 1995.

[43] L. Smolin. Strings as perturbations of evolving spin-networks. hepth/9801022.

[44] D.R. Finkelstein. Quantum Relativity: A Synthesis of the Ideas of Einstein and Heisenberg. Springer-Verlag, New York, 1996. 
[45] C.J. Isham and J. Butterfield. A topos perspective on the KochenSpecker theorem: I. Quantum states as generalised valuations. International Journal of Theoretical Physics, 37:2669-2733, 1998. quant$\mathrm{ph} / 980355$.

[46] J. Butterfield and C.J. Isham. A topos perspective on the KochenSpecker theorem: II. Conceptual aspects, and classical analogues. International Journal of Theoretical Physics, 1999. To appear. quant$\mathrm{ph} / 9808067$. 